\title{
Scattering frequencies and Gevrey 3 singularities
}

\author{
C. Bardos ${ }^{1,2}$, G. Lebeau ${ }^{1,3}$, and J. Rauch ${ }^{1,4}$ \\ ${ }^{1}$ C.M.A., ENS Ulm, 45, rue d'Ulm, F-75005 Paris, France \\ ${ }^{2}$ C.S.P., Université de Paris 13, Av. J.B. Clément, F-93430 Villetaneuse, France \\ ${ }^{3}$ Département de Mathématiques, Université de Paris Sud, Bâtiment 425, F-91405 Orsay, France \\ ${ }^{4}$ Mathematics Department, University of Michigan, Ann Arbor, MI 48109, USA
}

\section{Introduction}

In this paper we study the scattering frequencies $\mu_{k}$. For the Laplacian $\Delta$, in the region $\Omega=\mathbb{R}^{n} \backslash \mathcal{O}$ exterior to a compact analytic obstacle $\mathcal{O}$. We suppose that $n$ is odd and that Dirichlet boundary conditions are imposed on $\partial \Omega$. The set $\left\{\mu_{k}\right\}$, discrete in $\operatorname{Re} \mu<0$, arises in a variety of different disguises (see [L.P. 2]).

(1) They are the poles of the analytic continuation of the resolvent $\left(z^{2}-\Delta\right)^{-1}$ which is analytic for $\operatorname{Re} z>0$.

(2) They are the values $\mu$ such that the boundary value problem

$$
\left(\mu^{2}-\Delta\right) u=0 \quad \text { in } \Omega, \quad u=0 \quad \text { in } \partial \Omega,
$$

where $u$ satisfies the Sommerfeld radiation condition, has a non zero solution.

(3) Via an expansion $\sum e^{\mu} k^{k} u_{k}$, they describe the asymptotic behavior as $t \rightarrow \infty$ of solutions to

$$
\begin{gathered}
\partial_{t}^{2} u-\Delta u=0 \quad \text { in } \quad \mathbb{R}_{t} \times \Omega, \quad u=0 \text { in } \mathbb{R}_{t} \times \partial \Omega, \\
u(0, \cdot), u_{t}(0, \cdot) \text { of compact support. }
\end{gathered}
$$

(4) They are the poles of the analytic continuation of the scattering matrix.

(5) They form the spectrum of the infinitesimal generator $B$ of the Lax-Phillips semigroup $Z(t)$.

Because of (3) and (4) a particularly important role is played by those $\mu$ with small real part, and this paper addresses the question of how fast $\operatorname{Re} \mu$ must grow as $|\mu| \rightarrow \infty$.

Lax and Phillips recognized that the response is related to the propagation of singularities for (1.1). Their reasoning together with the resolution of the problem of propagation of $C^{\infty}$ singularities by Taylor, Melrose and Sjöstrand showed that for non trapping obstacles there is a constant $C>0$ such that:

$$
\operatorname{Re} \mu_{k} \leqq-C \log \left|\mu_{k}\right| \forall k \text {. }
$$


The key to this is the fact that solutions to (1.1), (1.2) are, for large $t, C^{\infty}$ on compact sets. Lebeau [L.2] has recently shown that for analytic, non trapping obstacles this can be improved to Gevrey 3. As in the earlier work of Goodhue [G], this allows us to improve the bound to

$$
\operatorname{Re} \mu_{k} \leqq-C\left|\mu_{k}\right|^{1 / 3} \forall k .
$$

For the case $\mathcal{O}=(|x| \leqq R)$, it can be shown by explicit calculation that there is an infinite number of $k$ with $\operatorname{Re} \mu_{k} \geqq-C\left|\mu_{k}\right|^{1 / 3}$ so, in that case, the bound (1.4) is optimal. Our main result is that this optimality is valid for most analytic strictly convex obstacle. In broadest terms, our idea is to associate resonances with a closed non degenerate geodesic on $\partial \Omega$. Such a geodesic is an "analytic ray" in the sense of Sjöstrand and is therefore the carrier of a function of class exactly Gevrey 3 which remains trapped. To take advantage of this, we follow [L.P.2] and [B.G.R.] (and also [D.G.] and [C]) in considering:

$$
\operatorname{Tr}(t)=\operatorname{Tr} Z(t)=\sum e^{\mu_{k} t},
$$

where the $\mu_{k}$ are repeated according to their algebraic multiplicity. When $\mathcal{O}$ is analytic and non trapping $\operatorname{Tr}$ is Gevrey 3 on $(0, \infty)$ and for strictly convex $\mathcal{O}$ we show that $\operatorname{Tr}$ is analytic except at the numbers $T \in \mathscr{L}$, the union of the lengths of closed geodesics in $\partial \Omega$. If $T$ is isolated in $L$ and is the length of exactly one geodesic which is, in addition, non degenerate, we obtain a lower bound

$$
\left|\partial_{t}^{n} T(t)\right| \geqq A C^{n}(3 n) ! \quad A, C>0,
$$

and it follows that with a constant $C^{\prime}>0$

$$
\#\left\{\mu_{k}: \operatorname{Re} \mu_{k} \geqq-C^{\prime}\left|\mu_{k}\right|^{1 / 3}\right\}=\infty
$$

showing that the bound (1.3) is sharp. In fact our results are more precise than (1.6), (1.7). And finally it is reasonable to expect that the convexity can be replaced by convexity near the closed geodesic.

The paper is organized as follows. In the first section, we state precisely our three main theorems corresponding to the bound (1.3), the regularity assertions about $\operatorname{Tr}(t)$ which we call the Poisson relation, and the assertion (1.7). We prove the first and then show how the last follows from the Poisson relation. We then show how the study of $\operatorname{Tr}$ can be reduced to the analysis of the restriction to $\mathbb{R}_{t} \times \partial \Omega$ of elementary solutions in $\mathbb{R}_{t} \times \mathbb{R}^{n}$ and $\mathbb{R}_{t} \times \mathcal{O}$. The crux of our proof is a calculation of the constant $C$ which appears in the Gevrey 3 regularity of $T r$. This constant can be viewed as a measure of regularity within the class Gevrey 3 and it is related to the Gevrey 3 regularity of solutions to (1.1). In Sect. II, we recall the Fourier Bros Iagolnizer characterizations of microlocal regularity. The notions of Gevrey 3 Lagrangian distributions and finally, Lagrangian distributions Gevrey 3 with weight $\psi$. The weight $\psi$ is a microlocal version of $C$. Section 3 (resp. 4) are devoted to a precise analysis of the elementary solution in $\mathbb{R}_{t} \times \mathbb{R}^{n}$ (resp. $\mathbb{R}_{t} \times \Omega$ ) as Lagrangian distributions. This done, the Poisson relation follows. The appendices contain two geometric results. The first computes a Maslov index and the second shows that, generically, obstacles do have closed geodesics with the properties we 
require. The results contained in this paper were announced in [B.L.R.]. By a different method (estimation of Greens functions) G. Popov [P.] has obtained upper bounds like (1.4).

\section{Main theorems}

Suppose $n \geqq 3$ is odd and that $\mathcal{O} \subset \mathbb{R}^{n}$ is a compact set and $\Omega=\mathbb{R}^{n} \backslash \mathcal{O}$. We assume that $\Omega$ is an analytic connected manifold with boundary $\partial \Omega$. Let $u$ be the solution of the wave equation with Dirichlet boundary conditions (1.1) and initial data

$$
u(0, \cdot)=u_{0}(\cdot), \quad \partial_{t} u(0, \cdot)=u_{1}(\cdot) .
$$

We denote by $\underline{u}(t) \equiv\left(u(t, \cdot), \partial_{t} u(t, \cdot)\right)$ the Cauchy data at time $t$ and by $H(\Omega)$ the completion of $C_{0}^{\infty}(\Omega) \times C_{0}^{\infty}(\Omega)$ in norm

$$
\left\|\left(u_{0}, u_{1}\right)\right\|^{2} \equiv\left|\left(u_{0}, u_{1}\right)\right|_{E}^{2}=\int_{\Omega}\left|D_{x} u_{0}\right|^{2}+\left|u_{t}\right|^{2} d x .
$$

Then, $H(\Omega)$ is a Hilbert space and the map $\underline{u}(t) \equiv U(t) \underline{u}(0)$ defines a unitary group with generator.

$$
A=\left[\begin{array}{ll}
0 & 1 \\
\Delta & 0
\end{array}\right]
$$

With $\varrho$ chosen so that $\mathscr{O}\left\{\{|x|<\varrho\}\right.$ we introduce the spaces $D_{ \pm}^{\varrho \perp}$ Lax-Phillips [L.P.1]:

$$
D_{ \pm}^{\varrho}=\{\underline{u} \in H: U(t) \underline{u}=0 \quad \text { if } \quad|x| \leqq \pm t+\varrho \text { and } \pm t>0\} .
$$

Then $D_{ \pm}^{e}$ are orthogonal, and if $P_{ \pm}^{Q}$ are the orthogonal projections on $D_{ \pm}^{e_{\perp}}$ then:

$$
Z(t) \equiv P_{+}^{\varrho} U(t) P^{\varrho}
$$

is a contraction semigroup on $K \equiv\left(D_{+}^{e} \oplus D_{-}^{e}\right)^{\perp} . Z$ describes the behavior of $U$ near $(\mathcal{O}$ in the sense that if $f \in H, \operatorname{supp} f \subset\{|x| \leqq \varrho\}$ then for all $t>0$

$$
Z(t) f=U(t) f \quad \text { on } \quad\{|x| \leqq \varrho\} .
$$

The infinitesimal generator, $B$, of $Z(t)$ has discrete spectrum in $\operatorname{Re} \mu<0$ consisting of eigenvalues of finite multiplicity. As discussed in the introduction (1.3) holds for non trapping obstacles (see [M.1] for definition).

Theorem 1. If the analytic manifold, $\partial \Omega$, is non trapping, then there is a constant $C>0$ such that

$$
\sigma(B) \subset\left\{\left.\mu \in \mathbb{C}|\operatorname{Re} \mu \leqq-c| \mu\right|^{1 / 3}\right\} .
$$

Our main result is that this bound is optimal for convex analytic manifolds satisfying an additional geometric hypothesis which is generically satisfied (see Appendix 2). 
In the Theorem 2, as in many problems of diffraction, the Airy function, $\mathrm{Ai}$, intervenes. The Fourier transform of $\operatorname{Ai}(x)$ is $\exp \left(i \xi^{3} / 3\right)$ and a brief discussion of its properties can be found on pages 203-205 of [H.2].

Theorem 2. Suppose that the Obstacle $\mathcal{O}$ is convex with analytic boundary of strictly positive curvature. Suppose that $\gamma$ is a geodesic or length $T$ on $\partial \mathcal{O}$ and satisfies

(1) There is a neigborhood of $T$ in $\mathbb{R}$ such that $\gamma$ is the only closed geodesic on $\partial \mathcal{O}$ whose length is in that neigbourhood.

(2) $\gamma$ is non degenerate in the sense that the first return map of the geodesic flow at time $T$ has 1 as a simple eigenvalue.

Then for any $\varepsilon>0$, the set

$$
\left\{\left.\mu \in \sigma(B)\left|\operatorname{Re} \mu \geqq-\left(C_{\gamma}+\varepsilon\right)\right| \mu\right|^{1 / 3}\right\}
$$

is infinite, where

$$
C_{\gamma} \equiv \omega \cos (\pi / 6)\left(2^{1 / 3} T\right)^{-1} \int_{0}^{T} \varrho^{2 / 3}(s) d s,
$$

$-\omega$ is the zero of smallest modulus of the Airy function, $\varrho$ is the curvature in $\mathbb{R}^{n}$ of $\gamma$, and $s$ is the arclength parameter.

Theorem 2 is a direct consequence of Melrose's [M.2] polynomial bound for the resonances and the following Poisson type relation in the Gevrey category.

Theorem 3 (Poisson Relation). Suppose that $\partial \Omega$ is analytic and that $\mathcal{O}$ is strictly convex. Then:

(i) The distribution $\operatorname{Tr}(\cdot)$ defined in (1.5) lies in $\left.G^{3}(10, \infty]\right)$.

(ii) If $L$ is the set of lengths of closed geodesics on $\partial \Omega$, then $\operatorname{Tr}$ is analytic on ] $0, \infty] \backslash L$.

(iii) If $T \in \mathscr{L}$ and $T$ is the length of exactly one closed geodesic $\gamma$ which in addition, is nondegenerate, then for any $\varepsilon>0$, one has:

$$
\limsup _{n \rightarrow \infty}\left[\sup _{|t-T|<\varepsilon}\left|((3 n) !)^{-1} \partial_{t}^{n} \operatorname{Tr}(t)\right|^{1 / 3 n}\right] \geqq\left(C_{\gamma} T\right)^{-1} .
$$

with $C_{\gamma}$ defined in (2.4).

In the remainder of this section, we derive Theorem 1 from Lebeau's $G^{3}$ propagation theorem and we deduce Theorem 2 from Theorem 3 and Melrose's polynomial bound. We then derive a representation for $\operatorname{Tr}(\cdot)$. The remaining sections are devoted to the analysis of the terms entering in this representation, culminating in a proof of Theorem 3.

Definition 2.1. For $\Omega \subset \mathbb{R}^{n}$ open, $s \in\left[1, \infty\left[\right.\right.$ and $f \in C^{\infty}(\Omega)$, we say that $f$ is of class Gevrey $s, f \in G^{s}(\Omega)$ symbolically, if for any compact $K \subset \Omega$, there are two constants $A_{\mathrm{K}}, B_{\mathrm{K}}$ such that

$$
\left(\forall \alpha \in \mathbb{N}^{n}\right) \sup _{K}\left|\partial_{x}^{\alpha} f\right| \leqq A_{K}\left(B_{K}\right)^{|\alpha|}(|\alpha| !)^{s} .
$$

The notion of $G^{5}$ on an open subset of a $C^{\omega}$ manifold or $C^{\omega}$ manifold with boundary is similar. In particular, $G^{1}(\Omega)$ is the space of real analytic functions on $\Omega$. 
For a distribution $f$, the set of points $p$ with the property that $f$ is Gevrey $s$ on a neighborhood of $p$ is open and its complement is called the Gevrey $s$ singular support.

Proof of Theorem 1. For any $R>\varrho$, let $\Omega_{R} \equiv \Omega \cap\{|x| \leqq R\}$ and consider Cauchy data $\underline{u}(0) \in H(\Omega)$ with supp $\underline{u} \in \Omega_{R}$. If $\mathcal{O}$ is nontrapping, then we know that there is a $t_{1}>0$ so that any " $C$-ray" beginning in $\Omega_{R}$ lies in $\Omega \cap\{|x|>R\}$ for $t>t_{1}$. By [L.3] we know that the Gevrey 3 singular support is a union of $C^{\infty}$ rays. Therefore the solution $\underline{u}(x, t)$ with Cauchy data $\underline{u}(0)$ is Gevrey 3 on $\left[t \geqq t_{1}\right] \cap \Omega_{R}$. Following a proof of Lax [L, Thm. 2.2] we show that this implies that there are constants $A, C$ depending only in $\Omega, t_{1}, t_{2}, R$ so that

$$
\forall 1 \geqq 0 \sup _{\left[t_{1}, t_{2}\right] \times \Omega_{R}}\left[\left|\partial_{t}^{1} u\right|+\left|\partial_{s}^{1} \nabla u\right|\right] \leqq A C^{1}(31) !\|u(0)\|_{E} .
$$

Toward this end let $\Gamma=\left[t_{1}, t_{2}\right] \times \Omega_{R}$ and, for $N \in \mathbb{N}$

$$
G_{N}^{3}=\left\{u \in C^{\infty}(\Gamma): \sup _{\Gamma, \alpha}\left|D^{\alpha} u\right| /(\alpha !)^{3} N^{|\alpha|}\right\}<\infty .
$$

Then $G_{N}^{3}(\Gamma)$ is a Banach space and $G^{3}(\Gamma)=U_{N} G_{N}^{3}(\Gamma)$. Let $A_{N} C H$ be the set of initial data with the property that the solution with data in $A_{N}$ lies in $G_{N}^{3}(\Gamma)$. Then $A_{N}$ is a linear subspace of $H$ and $U_{N} A_{N}=H$. The Baire Category theorem implies that for some $N_{0}, A_{N_{0}}$ is of the second category. The map $\left.H \supset A_{N_{0}} \ni \underline{u} \rightarrow u\right|_{\Gamma} \in G_{N_{0}}^{3}(\Gamma)$ is defined on a subspace of second category and has closed graph, hence is continuous, proving (2.6) with $C=N_{0}$.

Choose $\chi \in C^{\infty}$ with $\chi=0$ on $[2, \infty]$ and $\chi=1$ on $[-\infty, 1] . Q_{r}$ is then the truncation operator defined by $Q_{r} f(x)=\chi(|x| / r) f(x)$. Following Lax and Phillips: [L.P.1], let $M \equiv U(2 \varrho)-U_{0}(2 \varrho)$ where $U_{0}$ is the unitary group defined by the wave equation on $\mathbb{R}^{n}$ and $U(2 \varrho)$ is extended to $H\left(\mathbb{R}^{n}\right)$ as a linear map vanishing on $H(\Omega)^{\perp}$. Then formula (3.8) p. 155 of the above book asserts that for $t \geqq 4 \varrho$

$$
Z(t)=P_{+}^{e} M Q_{5 e} U(t-4 \varrho) Q_{3_{e}} M P_{-}^{Q} .
$$

Then for $t_{0} \geqq 4 \varrho$, and any $l \in \mathbb{Z}_{+}$,

$$
B^{l} Z\left(t_{0}\right)=\partial_{t}^{l} Z\left(t_{0}\right)=P_{+}^{e} M Q_{5 e} \partial_{t}^{l} U\left(t_{0}-4 \varrho\right) Q_{3 e} P_{-}^{e} .
$$

Choosing $R=10 \varrho$ and $t_{0}>t_{1}$, we see that (2.6) and (2.7) show that $B^{l} Z\left(t_{0}\right)$ is bounded from $H(\Omega)$ to itself with

$$
\left\|B^{l} Z\left(t_{0}\right)\right\| \leqq A^{\prime}\left(C^{\prime}\right)^{l}(3 l) ! \forall l .
$$

For $\mu \in \sigma(B), \mu^{l} e^{\mu t_{0}} \in \sigma\left(B^{l} Z\left(t_{0}\right)\right.$ and is therefore bounded by the norm of that operator:

$$
|\mu|^{1} e^{t_{0} \operatorname{Re} \mu} \leqq A^{\prime}\left(C^{\prime}\right)^{1} \cdot(3 l) !
$$

Given $\mu$, choose $l$ so that $\left|3 l-\left(|\mu| / C^{\prime}\right)^{1 / 3}\right|<1$ and use Stirling's formula to prove (2.2).

Proof of Theorem 2 assuming Theorem 3. We show that if $\sigma(B) \cap\left\{\operatorname{Re} \mu \geqq-C|\mu|^{1 / 3}\right\}$ is finite, then $C \leqq C_{\gamma}$. To accomplish this, we use the supposed finiteness and 
Melrose's polynomial bound [M.2]

$$
\exists_{N, M} \text { s.t. } \forall R \#\left\{\mu_{k}:\left|\mu_{k}\right| \leqq R\right\} \leqq M(R+1)^{N}
$$

to derive upper bounds for $\partial_{t}^{n} \operatorname{Tr}(t)$ which are then compared with (2.5). In the following sums, we sum such $\mu \in \sigma(B)$ repeated according to multiplicity. Let $M$ $C \sigma(B)$ be the finite set of $M$ with $|\mu| \leqq 1$ or $\operatorname{Re} \mu \geqq-C|\mu|^{1 / 3}$. Write

$$
\operatorname{Tr}(t)=\sum_{\mu \in M} e^{\mu t}+\sum_{l=1}^{\infty}\left(\sum_{l \leqq|\mu|<l+1} e^{\mu t}\right) \equiv \sum_{l=0}^{\infty} f_{1}(t) .
$$

Then $f_{0} \equiv \sum_{\mu \in M} e^{\mu t}$ is entire analytic and

$$
\left|\partial_{t}^{n} f_{1}\right| \leqq M(l+1)^{n+N} e^{-C l^{1 / 3 t}} .
$$

Thus, comparing the sum to an integral of a function with one maximum on $(0, \infty)$ yields

$$
\begin{aligned}
& \left|\partial_{t}^{n}\left(\operatorname{Tr}(t)-f_{0}(t)\right)\right| \leqq \sum_{t=1}^{\infty} M(l+1)^{n+N} e^{-C l^{1 / 3} t} \leqq M e^{c t} \int_{10, \infty[} x^{n+N} e^{-C t x^{1 / 3}} d x \\
& \left.+e^{c t} M \operatorname{Max}_{[0, \infty]}\left(x^{n+N} e^{-C t x^{1 / 3}}\right)\right) \leqq \text { const. }(C t)^{-3 n-3 N-3}(3 n+3 N+2) ! e^{c t}
\end{aligned}
$$

Thus, $\operatorname{Tr} \in G^{3}(0, \infty)$ and $\limsup _{n \rightarrow \infty}\left|(3 n !)^{-1} \partial_{t}^{n} \operatorname{Tr}(t)\right|^{1 / 3 n} \leqq(C t)^{-1}$. Taking $t=T$ and comparing with the conclusion (2.5) of Theorem 3, we see that we must have $C \leqq C_{\gamma}$.

In the remainder of this section, we will derive a representation formula for $\operatorname{Tr} Z(t)$. We begin with a formula of Melrose [M.2]

$$
\operatorname{Tr} Z(t)=2 \partial_{t} \int_{\Omega} E(t, x, x) d x, \quad t>0,
$$

where $E$ is the fundamental solution of $\square$ on $\mathbb{R}_{t} \times \Omega$, that is for $y \in \Omega$

$$
\begin{array}{rl}
\left(\partial_{t}^{2}-\Delta_{x}\right) E(t, x, y)=0 & \left.E(t, x, y)\right|_{(t, x) \in \mathbf{R}_{t} \times \partial \Omega}=0, \\
E(0, x, y)=0 & E_{t}(0, x, y)=\delta(x-y) .
\end{array}
$$

There are similar kernels $K^{ \pm}(t, x, y),(t, x, y) \in \mathbb{R} \times \Omega \times \partial \Omega$ associated with inhomogeneous Dirichlet conditions

$$
\left(\partial_{t}^{2}-\Delta_{x}\right) K^{ \pm}(t, x, y)=\left.0 \quad \operatorname{Supp} K^{ \pm} C\{ \pm t \geqq 0\} \quad K^{ \pm}(t, x, y)\right|_{(t, x) \in \mathbf{R}_{t} \times \partial \Omega}=\delta_{t=0, x=y} .
$$

Then

$$
F(t, x)=\int_{\mathbf{R} \times \partial \boldsymbol{\Omega}} K^{+}(t-s, x, y) f(s, y) d s d y
$$

is the solution of $\square F=0$, equal to $f$ on $\mathbb{R}_{t} \times \partial \Omega$ and vanishing for $t \leqq \min \{s:(\exists x)(s, x) \in \operatorname{supp} f\}$.

Define $k^{ \pm}(t, x, y) \in \mathscr{D}^{\prime}(\mathbb{R} \times \partial \Omega \times \partial \Omega)$ by

$$
K^{ \pm}=\left.\partial_{n_{x}} K^{+}\right|_{\mathbf{R}_{t} \times \partial \Omega \times \partial \Omega} .
$$


Then for $t, x \in \mathbb{R} \times \partial \Omega$,

$$
\partial_{n_{x}} F(t, x)=\iint k^{+}(t-s, x, y) f(x, y) d s d y
$$

so that $k^{+}$is the kernel of the Neumann operator. Similar formulas hold for $k^{-}$and for $f_{ \pm} \in \mathscr{E}(\mathbb{R} \times \partial \Omega)$, we can consider $F_{ \pm}$defined using $k^{ \pm}$. Green's identity applied to these functions yields

$$
\iint_{\partial \Omega \times \mathbb{R}}\left(F_{+} \partial_{n_{x}} F_{-}-\partial_{n_{x}} F_{+} F_{-}\right) d t d x=0 .
$$

As this holds for arbitrary boundary data $f_{ \pm}$, we have the symmetry relation

$$
k^{+}(t, x, y)=k^{-}(-t, y, x) \text {. }
$$

Finally we denote by $e_{0}^{+} \in \mathscr{D}^{\prime}\left(\mathbb{R} \times \mathbb{R}^{n}\right)$ the forward fundamental solution for $\square$, $\square e_{0}^{+}=\delta$, supp $e_{0}^{+} \subset\{t \geqq 0\}$.

Restricting $e_{0}^{+}(t, x-y)$ to $\mathbb{R} \times \Omega \times \Omega$ yields $E$ apart from a correction for the inhomogeneous Dirichlet data $\left.e_{0}^{+}(t, x-y)\right|_{(t, x) \in \mathbb{R} \times \partial \Omega} \in \mathscr{E}\left(\mathbb{R}_{t} \times \partial \Omega\right)$ :

$$
\begin{gathered}
E(t, x, y)=e_{0}^{+}(t, x-y)-E(t, x, y), \\
E(t, x, y) \equiv \int_{\mathbb{R} \times \partial \boldsymbol{\Omega}} K^{+}(t-s, x, z) e_{0}^{+}(s, z-y) d s d z .
\end{gathered}
$$

From Huyghen's principle, we see that $e_{0}^{+}(t, x-y)=0$ on a neighborhood of $x=y$ when $t>0$, so for $t>0$

$$
-\int_{\Omega} E(t, x, x) d x=\int_{\Omega} \widetilde{E}(t, x, x) d x, \quad=\int_{\Omega}\left(\int_{\mathbb{R} \times \partial \Omega} K^{+}(t-s, x, z) e_{0}^{+}(s, z-x) d s d z\right) d x .
$$

Introduce $h_{0}^{+}(t, x)$ solution in $\mathbb{R} \times \mathbb{R}^{n}$ to $\square h_{0}^{+}=e_{0}^{+}$, supp $h_{0}^{+} \subset\{t \geqq 0\}$. Then continuing the equality (2.11),

$$
=\int_{\partial \Omega} \int_{\Omega} \int_{\mathbb{R}} K^{+}(t-s, x, z)\left(\square h_{0}^{+}\right)(s, z-x) d s d x d z .
$$

Integration by parts in the $d s d x$ integral yields

$$
=\int_{\partial \Omega} \int_{\mathbb{R} \times \partial \Omega}\left(h_{0}^{+} \partial_{n_{x}} K^{+}-K^{+} \partial_{n_{x}} h_{0}^{+}\right) d s d x d z .
$$

Taking into account the boundary values of $K^{+}$, the second term is equal to $\int_{\partial \Omega} n_{x} \cdot \nabla_{x} h_{0}^{+}(t, 0) d x$. For $t>0, h_{0}^{+}(t, \cdot)$ is smooth near zero so $\nabla_{x} h_{0}^{+}(t, 0)$ is a fixed vector independent of $x \in \partial \Omega$ whence the above integral vanishes. Thus we have shown that

$$
-\operatorname{Tr} Z(t)=2 \partial_{t} \int_{\partial \Omega} B(t, x, x) d x
$$

where

$$
B(t, x, y) \equiv \int_{\mathbf{R}} \int_{\partial \Omega} h_{0}^{+}(t-s, z-x) k^{+}(s, z, y) d z d s
$$


The kernels that intervene here are the traces of the free propagation $h_{0}^{+}$and the Neumann operator $k^{+}$. The proof of Theorem 3 rests on a detailed analysis of these kernels in sections four and five respectively. The description uses the language of microlocal Gevrey regularity and Lagrangian distributions of class Gevrey 3 developed in Sect. 3.

\section{Fourier-Bros-Iagolnizer transform and conormal Gevrey distributions}

Following [S.1] we introduce the Fourier-Bros-Iagolnizer (F.B.I.) transform of $f \in S^{\prime}\left(\mathbb{R}^{n}\right)$ :

$$
T f(y, \lambda) \equiv \int e^{-\lambda(y-x)^{2} / 2} f(x) d x .
$$

Tf is defined on $\mathbb{C}^{n} \times \mathbb{R}_{+}$and is holomorphic with respect to $y$ and bounded by

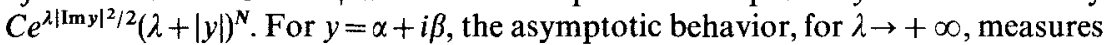
the microlocal regularity of $f$ at $(\alpha,-\beta) \in T^{*}\left(\mathbb{R}^{n}\right) \backslash\{0\}$. For example, it is not hard to show that

$$
\left(\alpha_{0}, \beta_{0}\right) \notin W F f \Leftrightarrow(\forall N>0)\left(\exists C_{N}\right) \text { s.t. }|T f(y, \lambda)| \leqq C_{N} e^{|\operatorname{lm} y|^{2} \lambda / 2} \lambda^{-N}
$$

holds uniformly for $y$ in a neighborhood of $\alpha_{0}-i \beta_{0}$ and $\lambda>1$. The Gevrey $s$ wave front set, $W F^{s}$, is measured similarly:

$$
\left(\alpha_{0}, \beta_{0}\right) \notin W F^{s} \Leftrightarrow(\exists C) \text { s.t. }|T f(y, \lambda)| \leqq A e^{\lambda|\operatorname{Im} y|^{2 / 2}} e^{-C \lambda^{1 / s}}
$$

for all $y$ in a neighborhood of $\alpha_{0}-i \beta_{0}$ and $\lambda>1$.

It is not obvious that the characterizations of $W F$ in terms of $T f$ are homogeneous with respect to the action of $\mathbb{R}_{+}$on the fibers. For the proof we recall Lebeau's inversion formula for $T$ [L.1]

$$
(2 \pi)^{d} f(x)=1 / 2 \int_{\mathbf{R}_{\xi}^{d}} e^{-R|\xi| / 2}\left(1-i\left(\xi /|\xi|^{2}\right) \cdot \nabla_{y}\right) T f(x-i R \xi /|\xi|,|\xi| / R) d \xi,
$$

where $R>0$ is at our disposal; then:

$$
T f(y, \lambda)=c \iint e^{-\lambda(y-x)^{2} / 2-R|\xi| / 2}\left(1-i\left(\xi /|\xi|^{2}\right) \cdot \nabla_{y}\right) T f(x-i R \xi /|\xi|,|\xi| / R) d \xi d x .
$$

The crucial fact is that for any $\delta>0$ the contribution from $|\xi / \lambda+\operatorname{Im} y|>\delta$ are dominated by $C e^{-\varepsilon \lambda} e^{\lambda|\operatorname{Im} y|^{2} / 2}$ for some $\varepsilon>0$. The contribution from $|\xi / \lambda+\operatorname{Im} y|<\delta$ is then estimated using (3.1). To see that $|\xi / \lambda+\operatorname{Im} y|>\delta$ is negligible, one deforms the $d x$ contour. Using the bound for $T f$ valid everywhere, the integrand has an exponential with exponent

$$
-\lambda(y-x)^{2} / 2-R|\xi| / 2+\left.|\operatorname{Im} x-R \xi /| \xi\right|^{2}|\xi| / 2 R
$$

which has real part

$$
-\lambda[\operatorname{Re}(y-x)]^{2} / 2+\lambda[\operatorname{Im} y]^{2} / 2-\operatorname{Im} x(\xi+\lambda \operatorname{Im} y)+(\operatorname{Im} x)^{2}(\lambda+|\xi| / R) / 2 .
$$

Deforming to $\operatorname{Im} x=\eta(\xi+\lambda \operatorname{Im} y) /(\xi \xi \lambda \operatorname{Im} y) \mid$ with $0<\eta \ll 1$ yields the desired result. 
We next recall Sjöstrand's [S1] intrinsic formulation. The holomorphic tangent space to $Y \equiv \mathbb{C}^{n}$ is spanned by $\partial / \partial y_{1}, \ldots, \partial / \partial y_{n}$, with

$$
\partial / \partial y_{j} \equiv 1 / 2\left(\partial / \partial \alpha_{j}-i \partial / \partial \beta_{j}\right), \quad \alpha_{j}=\operatorname{Re} y_{j}, \quad \beta_{j} \equiv \operatorname{Im} y_{j} .
$$

The holomorphic cotangent space is spanned by $d \zeta_{j}$ defined by $d \zeta_{j}\left(\partial / \partial y_{k}\right)=\delta_{j k}$ and the canonical two form $\sigma_{y}$ is defined by $\sum d \zeta_{j} \wedge d y_{j}$. Following Schapira [S.], we consider the $\mathbb{R}$ multilinear forms $\operatorname{Re} \sigma_{Y}$ and $\operatorname{Im} \sigma_{Y}$, with $d \gamma_{j} \equiv \operatorname{Re} d \zeta_{j}$ and $d \delta_{j} \equiv \operatorname{Im} d \zeta_{j}$ we have:

$$
\operatorname{Re} \sigma_{Y}=\sum_{j} d \gamma_{j} \wedge d \alpha_{j}-d \delta_{j} \wedge d \beta_{j}, \quad \operatorname{Im} \alpha_{Y}=\sum_{j} d \delta_{j} \wedge d \alpha_{j}+d \gamma_{j} \wedge d \beta_{j} .
$$

Both are symplectic forms on $T^{*} Y$, the latter viewed as a $C^{\omega}$ manifold of dimension $4 n$. (See [S.1, pp. 77-81].) If $\varphi$ is a real valued $C^{\omega}$ function on $Y$, then

$$
\Lambda_{\varphi} \equiv\left\{\left(y,-2 i \sum \partial \varphi / \partial y_{j}(y) \mid y \in Y\right\}\right.
$$

is a $C^{\omega}$ submanifold of $T^{*}\left(\mathbb{C}^{n}\right)$ of real dimension $2 n$ which is always $\operatorname{Im} \sigma_{Y}$ lagrangian. In addition $\Lambda_{\varphi}$ is $\operatorname{Re} \sigma_{Y}$ symplectic if and only if $\partial \partial \varphi$ is non degenerate. In this case $T^{*}\left(\mathbb{C}^{m}\right)$ is its complexification. This holds in particular if $\varphi$ is strictly plurisubharmonic (s.p.s.h.), that is

$$
\sum \partial^{2} \varphi / \partial y_{j} \partial \bar{y}_{k} w_{j} \bar{w}_{k}>0 \quad \forall w \in \mathbb{C}^{n} \backslash 0 .
$$

Suppose $M$ is a $C^{\omega}$ manifold of real dimension $n, p_{0} \in T^{*} M \backslash 0, \varphi \in C^{\omega}\left(\mathbb{C}^{n}\right)$ s.p.s.h., and $\chi$ a $C^{\omega}$ canonical transformation from a neighborhood of $p_{0}$ to $A_{\varphi}$ sending $p_{0}$ to the point of $A_{\varphi}$ over $y_{0}$. Denote by $X$ the complexification of $M$ and $\chi^{\mathbb{E}}$ the (local) holomorphic extension of $\chi$ to a map $T^{*} X \rightarrow T^{*} Y$. Then there is a unique holomorphic $g$ defined on a neighborhood of $\left(y_{0}, x_{0}\right)\left(x_{0} \equiv \pi\left(p_{0}\right)\right)$, such that

$$
g\left(y_{0}, x_{0}\right)=-i \varphi\left(y_{0}\right)
$$

(ii) The graph of $\chi^{\mathbb{C}}$ in $T^{*} X \times T^{*} Y$ is the set of points $(x, y,-\partial g / \partial x, \partial g / \partial y)$

(iii) The function $x \rightarrow-\operatorname{Img}(y, x)$ has a non degenerate critical point at

$$
x=\pi \chi^{-1}\left(y, \frac{2}{i} \frac{\partial \varphi}{\partial y}(y)\right) \text {. }
$$

The critical value is $\varphi(y)$ and the signature is $(0+, n-)$.

In particular, if $M=\mathbb{R}^{n}$ and $\chi$ is the mapping $(x, \xi) \rightarrow(x-i \xi, \xi)$ and if $\varphi$ is the function $\varphi(y)=1 / 2(\operatorname{Im} y)^{2}$, the function $g(x, y)$ which satisfies the properties (i), (ii), and (iii) is

$$
g(x, y)=i(x-y)^{2} / 2
$$

which appears in formula (3.1).

The above construction associates to any set $\left(M, \varphi, p_{0}, d x, \chi: T^{*} M \rightarrow \Lambda \varphi\right)$, an F.B.I. transform given by:

$$
\left(T_{\chi} f\right)(y, \lambda) \equiv \int e^{i \lambda g(y, x)} f(x) d x .
$$

In the right hand side, $d x$ is a $C^{\omega}$ volume element on $M, g$ is the function defined by the properties (i)-(iii); $\lambda$ belongs to $\mathbb{R}_{+}$and is assumed to be large; $f$ is a distribution (or more generally a hyperfunction) defined in a neighborhood of $x_{0}$. The function $T_{\chi} f$ is holomorphic with respect to $y$ and more precisely belongs to the $H_{\varphi}$ space of Sjöstrand, that is, for $y$ near $y_{0}$ it satisfies the estimate:

$$
\forall \varepsilon>0, \exists C_{\varepsilon}, \forall \lambda \geqq \lambda_{0}, \quad\left|T_{\chi} f(y, \lambda)\right| \leqq C_{\varepsilon} e^{\lambda(\varphi(y)+\varepsilon)} .
$$


The F.B.I. transform is used to characterize the microlocal structure of the analytic singular support according to the

Definition 3.1. For $s \in\left[1, \infty\left[\right.\right.$ and $f \in \mathscr{D}^{\prime}(M)$, the Gevreys wave front set, $G^{s} W F f$ $\subset T^{*} M \backslash 0$, is the complement of the set of points $p \in T^{*} M \backslash 0$ with the following property: there is an F.B.I. transform $T_{\chi}$ and a neighborhood $\omega$ of $\pi(\chi(p))$ such that

$$
(\exists C>0)(\forall z \in \omega, \lambda \geqq 1) \quad\left|T_{\chi} f(z, \lambda)\right| \leqq A e^{\lambda \varphi\left((z)-C \lambda^{1 / s}\right.} .
$$

Remarks. 1) The case $s=1$ yields the analytic wave front set

2) Functions Gevreys at $\pi(p)$ satisfy $\left|\partial_{x}^{\alpha} f\right| \leqq A B^{s|\alpha|}(s|\alpha|) \mid$. The constant $C$ in (3.4) is a microlocal analogue of the constant $1 / B$.

Lemma 3.1. The Definition (3.1) is homogeneous with respect to the $\mathbb{R}_{+}$action in the fibers. It is also independent of $\chi$ in the sense that (3.4) is satisfied for one $\chi$ iff it is satisfied for all.

Proof. If $\chi_{i}$ are canonical transformations on $\Lambda_{\varphi_{i}}, i=1,2$, then there exists a complex canonical transformation $\Gamma$ between the Sjöstrand spaces $H_{\varphi_{i}}$ with $\Gamma \circ T_{x_{2}}=T_{x_{i}}$. This yields the desired independence. The homogeneity is a consequence of the fact that the definitions are homogeneous for the ordinary F.B.I. as indicated at the beginning of this section.

In our analysis, it is important to know how the constant $C$ in (3.4) varies from point to point. This leads to the notion of Gevrey $s$ with weights. We consider $s=3$.

Definition 3.3. Suppose $m$ is a strictly positive function defined on a neighborhood of $p$ in $T^{*} M \backslash 0$ which is Lipschitz continuous, and homogeneous of degree $1 / 3$ in the fiber. A distribution $f \in \mathscr{D}^{\prime}(M)$ is microlocally Gevrey 3 with weight mat $p$ if for some F.B.I. transform $T_{\chi}$ there is a neighborhood $\omega$ of $\pi(\chi(p))$ such that $(\forall \varepsilon>)\left(\exists C_{\varepsilon}\right)$ $(\forall \lambda \geqq 1)(\forall y \in w)$

$$
\left|T_{\chi} f(y, \lambda)\right| \leqq C_{8} e^{\lambda \varphi(y)-\lambda^{1 / 3}(m(y)-\varepsilon)} \text {, where } m \equiv m \circ \pi_{Y} \circ \chi .
$$

Remarks. 1) That this is homogeneous and independent of $\chi$ follows as in Lemma 3.1.

2) Since $m>0$ Gevrey $^{3}$ with weight $m$ at $p$ implies Gevrey ${ }^{3}$ at $p$.

Next we define what is meant by a Lagrangian distribution of class Gevrey 3 . Suppose that $\Lambda \subset T^{*}(M)$ is a $C^{\omega}$ homogeneous Lagrangian submanifold and $\psi: \Lambda \rightarrow \mathbb{C}$ is $C^{\omega}$ and homogeneous of degree $1 / 3$ with $\operatorname{Re} \psi\left(p_{0}\right)>0$. Let $h(x, \theta)$ by a $C^{\omega}$ nondegenerate phase, which represents $A$ (see [H.1]). The parameter $\theta$ is defined in a conic neighborhood $\Gamma_{0}$ of $\theta_{0}$ in $\mathbb{R}^{N}$. Let

$$
C_{h} \equiv\left\{(x, \theta) \mid \partial_{\theta} h(x, \theta)=0\right\},
$$

and $j: C_{h} \rightarrow \Lambda$ the isomorphism defined by $j(x, \theta) \equiv\left(x, \partial_{x} h(x, \theta)\right)$ with $p_{0}=j\left(x_{0}, \theta_{0}\right)$. Let $l(x, \theta)$ be $C^{\omega}$, homogeneous of degree $1 / 3$ with $l_{C_{h}}=\psi \circ j$. We assume that $\Gamma_{0}$ is so small that $\operatorname{Re} l>0$.

Definition 3.4. A distribution $f \in \mathscr{D}(M)$ lies in $l_{\Lambda, \psi}^{3}$ at $p_{0}$ if for $x$ near $x_{0}=\pi\left(p_{0}\right)$, one has $f=f_{1}+f_{2}$ where $f_{2}$ is microlocally Gevrey ${ }^{3}$ with weight $m$ at $p_{0}$ such that $m\left(p_{0}\right)>\operatorname{Re} \psi\left(p_{0}\right)$ and

$$
f_{1}(x)=\int_{\Gamma_{0}} e^{i h(x, \theta)-l(x, \theta)} a(x, \theta) d \theta,
$$


where $a$ is an analytic symbol in the sense of Boutet de Monvel [BdM.] with an expansion

$$
a \sim \sum_{k=0}^{\infty} a_{k}(x, \theta),
$$

where the $a_{k}$ are $C^{\omega}$, homogeneous of degree $d-N / 2-k / 3$ in $\theta$. The expansion (3.7) means that there are constants $A, B$ and a complex conic neighborhood $N$ of $\pi\left(p_{0}\right)$ in $\mathbb{C}^{n} \times \mathbb{C}^{N}$ such that for all $x, \theta \in N$ and $k \in \mathbb{N}$

$$
\left|a-\sum_{k=0}^{k=K} a_{k}\right| \leqq A B^{K} K !|\theta|^{d-N / 2-(K+1) / 3} .
$$

Remarks. 1) Since Re $l>0$, the integral is absolutely convergent and $f_{1} \in G^{3}$.

2) This definition is clearly homogeneous in the fibers.

3) The fact that the definition is independent of the choice of $h$ and $l$ is proved in Proposition 3.1 below.

Preparing for Proposition 3.1, we introduce a canonical transformation $\chi$ as above and denote $\varphi_{A}$ the unique holomorphic function defined by (cf. [L.1]):

$$
\left(y, \frac{\partial \varphi_{\Lambda}}{\partial y}(y)\right)=\chi^{\mathbb{G}}\left(\Lambda^{\mathbb{G}}\right) ; \quad i \varphi_{\Lambda}\left(y_{0}\right)=\varphi\left(y_{0}\right) .
$$

Here, $\chi^{\mathbb{C}}, \Lambda^{\mathbb{C}}$ denote the natural complexifications. We recall that we have $-\operatorname{Im} \varphi_{\Lambda}(y) \leqq \varphi(y)$ and that equality holds if and only if $\left(y, \frac{2}{i} \frac{\partial \varphi_{\Lambda}}{\partial y}(y)\right) \in \chi(\Lambda)$. Let $\psi(y)$ be the holomorphic function on $Y$ defined by the relation: $\psi \circ \pi_{\gamma} \circ \chi^{\mathbb{C}}=\psi^{\mathbb{C}}$ where $\psi^{\mathbb{C}}$ denotes the holomorphic extension of $\psi$ to $A^{\mathbb{C}}$. Then we have the

Proposition 3.1. The following assertions are equivalent:

(i) $f$ belongs to $l_{A, w}^{3}$.

(ii) There exists a positive constant $\varepsilon$ such that, for $\left|y-y_{0}\right|<\varepsilon, T_{\chi} f$ admits the decomposition

$$
T_{\chi} f=e^{i \lambda \varphi_{A}(y)-\lambda^{1 / 3} \varphi(y)} b(y, \lambda)+r(y, \lambda),
$$

where the functions $r(y, \lambda)$ and $b(y, \lambda)$ have the following properties:

(a) $r(y, \lambda)$ satisfies the estimate

$$
|r(y, \lambda)| \leqq e^{\lambda \varphi(y)-C_{0} \lambda^{1 / 3}},
$$

with a constant $C_{0}$ strictly greater than $\operatorname{Re} \psi\left(p_{0}\right)$.

(b) $b(y, \lambda)$ is holomorphic with respect to $y$ and has, for $\left|y-y_{0}\right|<\varepsilon$ an asymptotic expansion of the form:

$$
\forall K \in \mathbb{N}, \sup _{\left|y-y_{0}\right|<\varepsilon}\left|b(y, \lambda)-\sum_{k=0}^{K} b_{k}(y) \lambda^{d-n / 2-k / 3}\right| \leqq A B^{K} K ! \lambda^{d-n / 2-(K+1) / 3} .
$$

Proof. First we prove that (i) implies (ii). Since $f_{2}$ is microlocally Gevrey 3 with weight $m$, the function $r(y, \lambda)=T_{\chi} f_{2}$ satisfies the estimate (3.5), and we have:

$$
T_{\chi} f_{1}=\lambda^{N} \int e^{i \lambda(g(y, x)+h(x, \alpha))-\lambda^{1 / 3} l(x, \alpha)} a(x, \lambda \alpha) d x d \alpha .
$$


Since $e^{-\lambda^{1 / 3} l(x, \alpha)} a(x, \lambda \alpha)$ is an analytic symbol, we can use standard contour deformations to reduce the problem to the use of the stationary phase method at the unique critical point of the mapping

$$
(x, \alpha) \rightarrow g(y, x)+h(x, \alpha)
$$

which is given by the relation

$$
(x, \alpha)=(x(y), \alpha(y))=j^{-1} \chi_{\mathbf{C}}^{-1}\left(y,\left(\partial_{y} \varphi_{A}\right)(y)\right) .
$$

The critical value is $\varphi_{A}(y)$, and we denote by $\psi(y)$ the expression $\psi(y)=l(x(y), \alpha(y))$. Using the variables of the Morse lemma, we have:

$$
T_{\chi} f_{1} \sim \lambda^{N} e^{i \lambda \varphi_{\Lambda}(y)-\lambda^{1 / 3} \psi(y)} \int_{|s| \leqq e} e^{-\lambda s^{2} / 2-\lambda^{1 / 3}(l(x(s), \alpha(s))-\psi(y))} a(y(s), \lambda \alpha(s)) J(s) d s .
$$

In this integral $J(s)$ denotes the Jacobian related to the change of variables. As it is well known we have, at the stationnary point

$$
\left.J(s)\right|_{s=0}=J(y, x(y), \alpha(y))=(\operatorname{det} \operatorname{Hess}(g+h) / i)^{-1 / 2},
$$

where the Hessian is taken with respect to both variables $x$ and $\alpha$ and where he square root is determined from the square of unity equal to 1 by the homotopy $s \rightarrow(1-s)$ Hess $(g+h) / i+s$ Id. A formal application of the method of the stationnary phase would give (see $[\mathrm{Sj} .1, \mathrm{p} .9])$

$$
T_{\chi} f_{1} \sim e^{i \lambda \varphi_{A}(y)-\lambda^{1 / 3} \hat{\varphi}(y)} \cdot \lambda^{(N-n) / 2} \sum_{k} \lambda^{-k} / k !(1 / 2 \Delta)^{k}\left(e^{-\lambda^{1 / 3}\{l(x, \alpha)-l(x(y), \alpha(y))} \cdot J a(x, \lambda \alpha)\right),
$$

where $\Delta$ is the Laplace operator in the variables from the Morse lemma.

To show that the above expression is not formal but satisfies indeed the estimates (3.10) we proceed as follow. (3.11), yields an integral of the form

$$
I=\int_{|s| \leqq e} e^{-\lambda s^{2} / 2+\lambda^{1 / 3} s F(y, s)} \sigma(y, s, \lambda) d s,
$$

where $\sigma$ is a classical analytical symbol in $\lambda^{-1 / 3}$. We split $I$ into two integrals $I_{1}$ and $I_{2}$ over $|s| \leqq \lambda^{-1 / 3} C_{0}$ and $\lambda^{-1 / 3} C_{0} \leqq|s| \leqq \varrho$ respectively. For $s \geqq \lambda^{-1 / 3} C_{0}$ we have $-\lambda s^{2} / 2+\lambda^{1 / 3} s|F| \leqq-\lambda^{1 / 3} C_{0}^{2} / 2+C_{0}|F|$ therefore, $I_{2}(y, \lambda)$ satisfies the estimate (3.9) (Note that $C_{0}$ can be taken arbitrarly large and that this type of estimate works for terms of the form $\lambda^{\alpha}$ only for $\alpha<1 / 2$.)

On the other hand, $I_{1}$ is given by the expression

$$
I_{1}=\int_{|t|<C_{0}} e^{-\lambda^{1 / 3} t^{2}} e^{t F\left(y, \lambda^{-1 / 3} t\right)} \sigma\left(y, \lambda^{-1 / 3} t, \lambda\right) \lambda^{-1 / 3} d t,
$$

which can be computed by the complex method of stationary phase with large parameter $\lambda^{1 / 3}$ which gives the same result as the formal expansion and which is of form (3.10).

To prove that (ii) implies (i), we use the microlocal nature of the problem; the property (i) is invariant under any real homogeneous canonical transform and (ii) is invariant under any canonical transform between Sjöstrand's spaces. We can assume that we have $A=T_{0}^{*} \mathbb{R}^{n}$ near $\left(0, \xi^{0}\right),\left|\xi^{0}\right|=1$ and that $T_{\chi}$ is the F.B.I. 
transform given by (3.1). In this situation, we have:

$$
\varphi(y)=1 / 2(\operatorname{Im} y)^{2}, \quad \varphi_{A}(y)=i y^{2} / 2 ; \quad \psi(y)=\psi(x=0, \xi=i y) .
$$

If we assume that (ii) is satisfied, there exists an analytical symbol (in the sense of Boutet de Monvel, i.e. holomorphic in $\lambda$ in a conic neighborhood of $\left.\mathbb{R}_{+}\right) \tilde{b}(y, \lambda)$ which has the same formal asymptotic expansion as $b(y, \lambda)$. Since we have, for $\lambda \geqq 1$, $|\tilde{b}-b| \leqq e^{-c \lambda^{1 / 3}}(c>0)$, we can replace $b$ by $\tilde{b}$, with only a change on the term $r(y, \lambda)$. Then we use the inversion formula, with $R=1$. We introduce a small conic neighborhood $\Gamma_{0}$ of $\xi_{0}$ and, microlocally near $\left(0, \xi_{0}\right)$, we can use $(3.8) ; f$ is microlocally the sum of the two following functions:

$$
\begin{gathered}
\left.f_{2}(x)=(1 / 2)(2 \pi)^{-n} \int_{I_{0}} e^{-|\xi| / 2}\left(1-i\left(\xi /|\xi|^{2}\right) \cdot \partial_{x}\right) r\right)(x-i \xi /|\xi|,|\xi|) d \xi, \\
f_{1}(x)=(2 \pi)^{-n} \int_{I_{0}} e^{i x \cdot \xi-\left(x^{2} / 2\right)|\xi|-|\xi|^{1 / 3} \psi(i x+\xi / \xi \xi)} \mathscr{L}(b) d \xi .
\end{gathered}
$$

The term $\mathscr{L}(b)$ is given by the formula:

$$
\mathscr{L}(b)=(1 / 2)\left[\left(1-i\left(\xi /|\xi|^{2}\right) \cdot \partial_{y}\right) b+i \xi /|\xi|^{2}\left(|\xi|(x-i \xi /|\xi|)+|\xi|^{1 / 3} \partial_{y} \hat{\psi}\right) b\right] .
$$

As in the proof of the Lemma 3.1, the function $f_{2}$ is microlocally Gevrey 3 with a weight $m$, satisfying the estimate $m\left(0, \xi^{0}\right)>\operatorname{Re} \psi\left(\xi^{0}\right)$. Finally, since $\mathscr{L}(b)(x, \xi)$ is a symbol in the sense of Boutet de Monvel, one can introduce, for $|x|$ small, the change of variables $\xi \rightarrow \xi+i(x / 2)|\xi|$, and obtain for $f_{1}$ an expression in terms of the real phase $x \cdot \xi$ corresponding to right hand side of (3.6). The proof of the Proposition (3.1) is complete.

\section{Traces of the fundamental solutions for $\square, \square^{2}$}

Our goal is to analyse $\operatorname{Tr}(t)$ using formulas (2.12) and (2.13). In $B$, two kernels intervene, $h_{0}^{+}$and $k^{+}$. The latter is the Neumann operator on $\mathbb{R}_{t} \times \Omega$ and the former is the trace on $\mathbb{R}_{t} \times \partial \Omega$ of a fundamental solution to $\square^{2}$ on $\mathbb{R} \times \mathbb{R}^{n}$. In this section, we study this second type of kernel.

The fundamental solutions $e_{0}, h_{0}$ are defined by:

$$
\square e_{0}^{+}=\delta, \quad \square^{2} h_{0}^{+}=\delta, \quad \operatorname{supp} e_{0}^{+}, h_{0}^{+} \subset\{t \geqq 0\} .
$$

In this section, we study the operators

$$
\begin{aligned}
& \mathbb{T}^{j}: \mathscr{E}^{\prime}\left(\mathbb{R}_{t} \times \partial \Omega\right) \rightarrow \mathscr{D}\left(\mathbb{R}_{t} \times \partial \Omega\right) \\
& \mathbb{T}^{1} f \equiv\left[e_{0}^{+} *\left(f \otimes \delta_{\partial \Omega}\right)\right]_{\mid \mathbb{R}_{t} \times \partial \Omega}
\end{aligned}
$$

and $\mathbb{T}^{2}$ is defined with $h_{0}^{+}$in place of $e_{0}^{+}$. Here $\partial \Omega$ is the boundary of the open set $\mathbb{R}^{n} \backslash 0$ and the traces are taken from the $\Omega$ side. From the definition, we see that the kernels of $T^{i}$ which are functions of $t, x, s, z \in\left(\mathbb{R}_{t} \times \partial \Omega\right)^{2}$ depend only on $t-s, x, z$ so 
we write as $\mathbb{T}^{i}(t-s, x, y)$. Again from the definition, we see that

$$
\begin{aligned}
& \mathbb{T}^{1}(t, x, z)=\left.e_{0}^{+}(t, x-z)\right|_{x \in \partial \Omega, z \in \partial \Omega}, \\
& \mathbb{T}^{2}(t, x, z)=\left.h_{0}^{+}(t, x-z)\right|_{x \in \partial \Omega, z \in \partial \Omega} .
\end{aligned}
$$

$\partial \Omega \times \mathbb{R}_{t}$ is non caracteristic for the wave equation and therefore, with the exception of $t=0$ and $x=z$ the trace of $e_{0}^{+}$and $h_{0}^{+}$on $\partial \Omega \times \mathbb{R}_{t}$ is well defined and we have:

$$
W F\left(\left.e_{0}^{+}\right|_{\partial \Omega X \partial \Omega X \mathbb{R}_{t}}\right) \subset \pi W F\left(e_{0}^{+}\right) \cap T^{*}\left(\partial \Omega X \partial \Omega X \mathbb{R}_{t}\right)
$$

and

$$
W F\left(\left.h_{0}^{+}\right|_{\partial \Omega X \partial \Omega X \mathbb{R}_{t}}\right) \subset \pi W F\left(h_{0}^{+}\right) \cap T^{*}\left(\partial \Omega X \partial \Omega X \mathbb{R}_{t}\right),
$$

where $\pi$ is the projection on $T^{*}\left(\partial \Omega X \partial \Omega X \mathbb{R}_{t}\right)$. The only difficulty for the definition of the trace arises near $t=0$ and $x=z=0$ where we will, with a change of variables, make an explicit computation. These computations are classical for elliptic problems and like in the elliptic case they give differents results when the trace is taken from the interior or from the exterior of the obstacle due to a change of sign in the determination of the square root.

We denote by $\widehat{\mathbb{T}}^{j}(\tau, x, z)$ the partial Fourier transforms with respect to $t$, so $\widehat{\mathbb{T}}^{j}$ is holomorphic in $\operatorname{Im} \tau<0$, and we have

$$
\mathbf{T}^{j}(\tau, x, y)=\left.(2 \pi)^{-n-1} \int\left(\xi^{2}-\tau^{2}\right)^{-j} e^{i(x-y) \xi} d \xi\right|_{x \in \partial \Omega, y \in \partial \Omega}, \quad \operatorname{Im} \tau<0 .
$$

If $x^{0} \in \partial \Omega$, we may relabel the coordinates so that near $x^{0}, \Omega$ is given by $\left\{x_{n}>S\left(x^{\prime}\right)\right\}$, where $x^{\prime} \equiv\left(x_{1}, \ldots, x_{n-1}\right)$. With $x^{\prime}$ as coordinates for $\partial \Omega$, the canonial projection $T_{x}^{*} \mathbb{R}^{n} \rightarrow T_{x}^{*} \partial \Omega$ for $x \in \partial \Omega$ is given by

$$
\xi^{\prime}, \xi_{n} \rightarrow \xi^{\prime}+\xi_{n} \nabla_{x^{\prime}} S\left(x^{\prime}\right) .
$$

Choose a $C^{\omega}\left(\mathbb{R}^{n-1}, \mathbb{R}^{n-1}\right)$ function $H$ such that

$$
S\left(x^{\prime}\right)-S\left(y^{\prime}\right)=\left(x^{\prime}-y^{\prime}\right) H\left(x^{\prime}, y^{\prime}\right), \quad H\left(x^{\prime}, x^{\prime}\right)=\partial_{x^{\prime}} S\left(x^{\prime}\right) .
$$

Then, the metric form $\Theta$ on $T^{*}(\partial \Omega)$ induced by the Euclidean metric is given by $\theta\left(x^{\prime}, x^{\prime}, \cdot\right)$ where

$$
\Theta^{2}\left(x^{\prime}, y^{\prime}, v^{\prime}\right)=v^{\prime 2}-\left(v^{\prime} \cdot H\left(x^{\prime}, y^{\prime}\right)^{2} /\left(1+H^{2}\right),\right.
$$

where the scalar products on the right are $\mathbb{R}^{n-1}$ Euclidean. Then $\Theta$ is a positive definite form on $T_{x^{\prime}}^{*}(\partial \Omega)$ provided $\left|y^{\prime}-x^{\prime}\right|$ is small. The glancing set is given by $\tau^{2}=\Theta^{2}\left(x^{\prime}, x^{\prime}, v^{\prime}\right)$, and the second fundamental form, $R$, by

$$
R\left(x^{\prime}, v^{\prime}\right)=-\operatorname{Hess} S\left(\xi^{\prime}, \xi^{\prime}\right) \cdot\left[\left(1+|\nabla S|^{2}+|\nabla S|^{4}\right) /\left(1+|\nabla S|^{2}\right)^{2}\right]^{1 / 2},
$$

where $\xi^{\prime}$ is defined by

$$
\xi^{\prime} \equiv v^{\prime}-\xi_{n} \nabla_{x^{\prime}} S, \quad \xi_{n} \equiv v^{\prime} \cdot \nabla S /\left(1+|\nabla S|^{2}\right) .
$$

The quadratic form $R$ gives the curvature in $\mathbb{R}^{n}$ of the $\partial \Omega$ geodesic passing through $\left(x^{\prime}, v^{\prime}\right)$ when $\Theta\left(x^{\prime}, x^{\prime}, v^{\prime}\right)=1$. 
In the coordinates $l, x^{\prime}, l \equiv x_{n}-S\left(x^{\prime}\right)$, we have $\delta_{\partial \Omega}=\left(1+\left|\nabla S\left(x^{\prime}\right)\right|\right)^{1 / 2} \otimes \delta(l)$. Thus (4.2) yields

$$
\hat{\mathbb{T}}^{j}(\tau, x, y)=(2 \pi)^{-n-1} \int e^{i \xi^{\prime}\left(x^{\prime}-y^{\prime}\right)+i \xi_{n}\left(x_{n}-S\left(x^{\prime}\right)\right)}\left(1+\left|\nabla S\left(y^{\prime}\right)\right|^{2}\right)^{1 / 2}\left(\xi^{2}-\tau^{2}\right)^{-j} d \xi .
$$

Following a calculation which is standard in the elliptic case, we take for new phase variables $v^{\prime}=\xi^{\prime}+\xi_{n} H\left(x^{\prime}, y^{\prime}\right), v_{n}=\xi_{n}$ and integrating with respect to $v_{n}$ and then letting $l=x_{n}-S\left(x^{\prime}\right)$ decrease to zero, we find that

$$
\hat{\mathbb{T}}^{j}\left(\tau, x^{\prime}, y^{\prime}\right)=2^{-j}(2 \pi)^{-n} \int e^{i v^{\prime}\left(x^{\prime}-y^{\prime}\right)}\left(\Theta^{2}-\tau^{2}\right)^{(1-2 j) / 2}\left[\left(1+\left|\nabla S\left(y^{\prime}\right)\right|^{2}\right) /\left(1+H\left(x^{\prime} y^{\prime}\right)^{2}\right)\right]^{1 / 2} d v^{\prime} .
$$

The square root of $\Theta^{2}-\tau^{2}$ is chosen with positive real part. Next, let $Q\left(x^{\prime}, y^{\prime}\right)$ be the positive definite matrix such that

$$
\Theta^{2}\left(x^{\prime}, y^{\prime}, v^{\prime}\right)=\left|Q^{-1} v^{\prime}\right|^{2}
$$

and introduce the new variable $\xi^{\prime} \equiv Q^{-1} v^{\prime}$ to find

$$
\widehat{\mathbb{T}}^{j}\left(\tau, x^{\prime}, y^{\prime}\right)=f\left(x^{\prime}, y^{\prime}\right) \int e^{i \xi^{\prime} \cdot Q\left(x^{\prime}, y^{\prime}\right)\left(x^{\prime}-y^{\prime}\right)}\left(\xi^{\prime 2}-\tau^{2}\right)^{\alpha_{j}} \cdot d \xi^{\prime} .
$$

Thus, the analytic spectrum of $T^{j}$ can be computed in terms of the inverse Fourier transform of the distribution $\left(\xi^{\prime 2}-(\tau-i 0)^{2}\right)^{\alpha_{j}}$ which is well known. In particular, the singular spectrum of $\mathbb{T}^{j}$ is, for $t>0$, the restriction to $\partial \Omega$ of the propagation for the wave equation; therefore we have the following:

Lemma 4.1. The analytic wave front of the kernels $\mathbb{T}^{j}(t, x, z)$ is contained in the set of the points $(t, x, z ; \tilde{t}, \tilde{x}, \tilde{z})$ which satisfy one of the two following relations

$$
t=0, \quad x=z, \quad \tilde{x}+\tilde{z}=0,
$$

or,

$$
t>0, \exists \xi \in \mathbb{R}^{n}, \quad \tilde{z}=-p_{z}(\xi), \quad \tilde{x}=p_{x}(\xi), \quad \tilde{t}=\mp t \xi /|\xi|, \quad x=z \pm t \xi /|\xi| .
$$

Here $p_{x}$ denotes the canonical projection of $T_{x}^{*}\left(\mathbb{R}^{n}\right)$ on $T_{x}^{*}(\partial \Omega)$.

Next, we use the results of Sect. 3 to study the propagation of Gevrey 3 singularities on $\partial \Omega$. Consider the $C^{\omega}, 2 n-1$ manifold $M \equiv \mathbb{R}_{t} \times \partial \Omega \times \partial \Omega$. In $T^{*} M$, we introduce the lagrangian manifold $A=A^{+} \cup A^{-}$where

$$
A^{ \pm}=\left\{(t, x, y ; \tau, \xi, \eta)| \pm \tau=| \xi|=| \eta \mid,(x, \xi)=\Phi_{+\tau}(y,-\eta)\right\} .
$$

The metric on $\partial \Omega$ is induced by the Euclidian metric in $\mathbb{R}^{n}$ and $\Phi$ denotes the geodesic flow, or more precisely the flow induced on $T^{*}(\partial \Omega)$ by the Hamiltonian $H_{|\xi|}$. We will denote by $\varrho(x, \xi)$ the curvature of the geodesic through the point $(x, \xi /|\xi|)$ and by $-\omega$ the zero of smallest modulus of the Airy function. Since $\tau+|\xi|=0$ on $A^{ \pm}$, we have $H_{t+|\xi|}$ tangent to $A^{ \pm}$and we define $\psi$ on $A^{ \pm}$by

$$
H_{\tau+|\xi|} \psi=e^{ \pm i \pi / 6}\left( \pm \tau \varrho^{2} / 2\right)^{1 / 3} \omega,\left.\quad \psi\right|_{t=0}=0 .
$$

Our analysis turns on the fact that several distributions entering in the description of $\operatorname{Tr} Z$ lie in $I_{A, \psi}^{3}$ and have leading terms which can be explicitly calculated. The two propositions of this section are results of that form. 
Proposition 4.1. If $f \in D^{\prime}\left(\mathbb{R}_{t} \times \partial \Omega \times \partial \Omega\right), \operatorname{supp} f \subset(t \geqq 0\}$, has analytic wave front set in the union $(t=0$ and $x=y) \cup(\Lambda \cap(t>0))$ and lies in $I_{A, \psi}^{3}$ for $t>0$ then on $a$ neighborhood of the set $(t>0,|x-z|<t)$ the distributions $\mathrm{g}^{j}$,

$$
\mathbf{g}^{j}(, x, z) \equiv \int_{\mathbb{R}_{s} \times \partial \Omega} \mathbb{T}^{j}(t-s, x, y) f(s, y, z) d s d y
$$

have analytic wave front set in $A$ and lie in $I_{A, \psi}^{3}$.

Proof. Since $\mathbb{T}^{j}$ propagates at speed exactly equal to one and $|x-z|<t$, the singularities of $f$ at $t=0$ do not influence those of $g(t, x, z)$. In addition, as $A$ lies in the glancing set and $\partial \Omega$ is strictly convex, singularities in $f$ for $t>0$ are propagated along glancing rays, and therefore away from $\partial \Omega$. This shows that the analytic wave front of $g^{k}$ is in $\Lambda$ and in addition that for $p_{0}=\left(t_{0}, x_{0}, z_{0}, \tau_{0}, \xi_{0}, \eta_{0}\right) \in A$ the microlocal behavior of $g^{j}$ near $p_{0}$ is determined by that of $f$ near $p_{0}$ and the microlocal behavior of $\mathbf{T}^{k}$ at $\left(0, x_{0}, x_{0}, \tau_{0}, \xi_{0},-\xi_{0}\right)$. For the rest of the proof, we suppose that $p_{0} \in \Lambda^{+}$. The case $p_{0} \in \Lambda^{-}$is similar. We compute in coordinates $x^{\prime}, x_{n}$ with $\Omega=\left\{x_{n}>S\left(x^{\prime}\right)\right\}$ as above. We write $f=f_{1}+f_{2}$ with $f_{2}$ Gevrey 3 with weight $m$, $m(p)>\operatorname{Re} \psi\left(p_{0}\right)$ and

$$
f_{1}(s, y, z)=\int e^{i \varphi(s, y, z, \theta)-t(s, y, z, \theta)} a(s, y, z, \theta) d \theta,
$$

where the phase $\varphi$ is defined by the eikonal equation on $\partial \Omega$ :

$$
\partial \varphi / \partial s=\Theta\left(y, y, \partial_{y} \varphi\right),\left.\quad \varphi\right|_{s=t_{0}}=\varphi_{0}(y, z, \theta),
$$

where $\varphi_{0}$ is a non degenerate phase describing $A^{+}$near $\exp \left(-t_{0} H_{|\xi|}\left(z_{0},-\eta_{0}\right)\right)$.

In the integrals (4.6) defining $\mathbb{T}^{k}(\tau, x, y)$, we can take the inverse Fourier transform to find expressions for $\mathbb{T}^{k}(t, x, y)$. In the resulting expression, there are two possible sources of trouble, the vanishing of the denominator when $\tau^{2}=\Theta^{2}$ and contributions from $\nu, \tau \rightarrow \infty, \tau^{2} \neq \Theta^{2}$. The latter is the transform of classical symbol and does not affect $T^{k}$ microlocally at $\left(0, x_{0}, x_{0}, \tau_{0}, \xi_{0},-\xi_{0}\right)$. Thus we may restrict attention to a neighborhood of $\tau^{2}=\Theta^{2}$ where we introduce the new variables $\sigma, v^{\prime}$ by $\tau=\Theta(x, y, v)(1-\sigma),|\sigma| \leqq \sigma_{0}$ small, and modulo a microlocally analytic correction we have:

$$
\mathbb{T}^{k}=\int_{-\sigma_{0}}^{\sigma_{0}}(\sigma+i 0)^{1 / 2-k} \int_{\Gamma_{0}} e^{i v \cdot(x-y)+(t-s)(1-\sigma) \boldsymbol{\theta}(x, y, v)} H^{k}(x, y, \sigma, v) d v d \sigma,
$$

where $\Gamma_{0}$ is a conic neighborhood of $\xi_{0}, H^{k} \in C^{\omega}$ is homogeneous of degree $2-2 k$ with respect to $v$, and we have suppressed the prime in $v$. Thus,

$$
\mathbb{T}^{k}=\int_{-\sigma_{0}}^{\sigma_{0}}(\sigma+i 0)^{+1 / 2-k} \mathbb{T}_{\sigma}^{k} d \sigma
$$

where $\mathbb{T}_{\sigma}^{k}$ are Fourier integral operators with canonical relations $C_{\sigma}$ determined by the phase $\Phi_{\sigma}(x, t, y, s, v) \equiv v \cdot(x-y)+(t-s)(1-\sigma) \Theta(x, y, v)$.

We do not need to take into account the boundary of $\Gamma_{0}$, because the point $\left(t_{0}\right.$, $\left.x_{0}, \tau_{0}, \xi_{0} ; t_{0}, x_{0}, \tau_{0}, \xi_{0}\right)$ does not belong to the canonical relation of $\Phi_{q \mid \partial \Gamma_{0}}$. Notice also that for $(t, s)$ near $\left(t_{0}, t_{0}\right), \Phi_{\sigma}$ induces a canonical isomorphism of $T^{*}(\partial \Omega)$ into itself, which is close to the identity. (The relation $C_{\sigma}$ itself is not an isomorphism 
but one can check that it induces a ramified canonical transformation with two sheets; the branching points occur at $\sigma=0$ along the glancing hypersurface $\mathscr{G}$.)

To compute $\left(\mathbb{T}^{k} f_{1}\right)(t, x, z)$, we superpose the integrals (4.11) and (4.12) and use the classical stationary phase method to eliminate the variables $(y, v)$. The critical points are given by the equations:

$$
\begin{aligned}
& y_{c}=x+(t-s)(1-\sigma) \partial_{v} \Theta\left(x, y_{c}, v_{c}\right) \\
& v_{c}=\partial_{y} \varphi\left(s, y_{c}, z, \theta\right)+(t-s)(1-\sigma) \partial_{y} \Theta\left(x, y_{c}, v_{c}\right) .
\end{aligned}
$$

We will denote by $v_{0}$ the vector $\partial_{x} \varphi(t, x, z, \theta)$. To compute the critical value, we assume that the function $S$ which describes $\partial \Omega$ near $x$, satisfies

$$
S(x+u)=-{ }^{t} u A u+O\left(|u|^{3}\right),
$$

where $A$ is a non degenerate positive symmetric matrix. In this situation, we have the relations:

$$
\Theta(x, x, v) \equiv|v|, \quad \partial_{x} \Theta(x, x, v) \equiv \partial_{y} \Theta(x, x, v) \equiv 0, \quad \partial_{v} \Theta=v /|v|
$$

and the Hessians $\partial_{x x}^{2} \Theta, \partial_{y y}^{2} \Theta, \partial_{x y}^{2} \Theta$ are all equal to $-1 /|v|\left({ }^{t} \cdot A v\right)^{2}$. Finally the curvature of the geodesic through $(x, v /|v|)$ is given by the relation

$$
\varrho(v, x)=2^{t} v \cdot A v /|v|^{2} .
$$

Using these formulas, we obtain the expression:

$$
\mathbb{T}^{k} f_{1}(t, x, z)=\int e^{i \varphi(t, x, z, \theta)-l(t, x, z, \theta)} b^{k}(t, x, z, \theta) d \theta,
$$

where the $b^{k}$ are given by the relations

$$
\begin{aligned}
\cdot b^{k}(t, x, z, \theta)= & \int_{1-\sigma_{0}, \sigma_{0} l}(\sigma+i 0)^{1 / 2-k} d \sigma \int e^{i g \theta\left(x, x, v_{0}\right)+l(t, x, z, \theta)} \\
& \times\left. Q\left(H^{k} a e^{-l(s, y, z, \theta)}\right)\right|_{y=y_{c}, v=v_{e}} d s .
\end{aligned}
$$

$Q$ denotes the operator from the asymptotic expansion in the stationary phase method; $H^{k}$ is the function defined by the formula (4.12) and $g \in C^{\omega}$ is homogeneous of degree zero with respect to $\theta$ with:

$$
g(s, t, x, z, \sigma, \theta)=\sigma(s-t)-(1 / 3)(1 / 8)(s-t)^{3} \varrho^{2}\left(x, v_{0}\right)+O\left((s-t)^{4}+\sigma(t-s)^{2}\right) .
$$

With (4.13), we have:

$$
\begin{aligned}
l(t, x, z, \theta)-l\left(s, y_{c}, z, \theta\right)= & (t-s)\left(\partial_{t} l(t, x, z, \theta)-\partial_{v} \Theta\left(x, x, v_{0}\right) \partial_{x} l(t, x, z, \theta)\right) \\
& +O\left((t-s)^{2}+\sigma(t-s)\right),
\end{aligned}
$$

and using (4.10), we obtain:

$$
\begin{aligned}
l(t, x, z, \theta)-l\left(s, y_{c}, z, \theta\right)= & (t-s)\left(\Theta\left(x, x, v_{0}\right)\right)^{1 / 3} \varrho^{2 / 3}\left(x, v_{0}\right) e^{i \pi / 6} 2^{-1 / 3} \omega \\
& +O\left((t-s)^{2}+\sigma(t-s)\right) .
\end{aligned}
$$

Finally, the relation (4.15) is valid mirolocally near $p_{0}$ if the integration with respect to $s$ in (4.16) is done in a neighborhood of $t_{0}$ which is small compared to $\sigma_{0}$, that is $\left|\sigma_{0}\right| \gg(t-s)^{2}$ (see $(4.17)$ ). 
We denote by $\lambda$ the expression $\Theta\left(x, x, v_{0}\right)$ and instead of $(\sigma, t-s)$, we introduce the new variables $(\hat{\sigma}, \hat{s})$ with the following properties: $(\hat{\sigma}, \hat{s})$ depend analytically on $z, \theta /|\theta|, t, x, \lambda^{-2 / 3}$, and satisfy the relations:

$$
\left.\hat{\sigma}\right|_{\sigma=0} \equiv 0,\left.\quad \hat{s}\right|_{t=s} \equiv 0, \quad \hat{s}>0
$$

if and only if $t>s$, and

$g(s, t, x, z, \theta)-i \lambda^{-2 / 3}\left[l(t, x, z, \theta)-l\left(s, y_{c}, z, \theta\right)\right]=-\hat{\sigma} \hat{s}+\hat{s}^{3} / 3-i \lambda^{-2 / 3} \hat{s} a(t, x, z, \theta /|\theta| ; \hat{s})$.

This reduces the study of the right hand side of $(4.15)$ to the asymptotic evaluations of the integrals:

$$
I_{k}(\lambda)=\iint_{C} e^{i \lambda\left(-\hat{\sigma} \cdot \hat{s}+\hat{s}^{3} / 3-i \lambda-2 / \hat{s} a(\hat{s})\right.} f(\hat{\sigma}, \hat{s}, \lambda)(\hat{\sigma}+i 0)^{1 / 2-k} d \hat{\sigma} d \hat{s},
$$

where $f$ denotes an analytic symbol in $\lambda$ which is classical in $\lambda^{1 / 3}$ and where $C$ is the $\mathbb{R}^{2}$ neighborhood of the critical point $(\sigma, s)=(0,0)$ defined by:

$$
|\hat{\sigma}| \leqq \hat{\sigma}_{0} ; \quad|\hat{s}| \leqq \hat{s}_{0} \quad \text { with } \quad \hat{\sigma}_{0} \gg \hat{s}_{0}^{2} .
$$

We denote by $\Phi$ the function

$$
\Phi(\hat{\sigma}, \hat{s})=-\hat{\sigma} \cdot \hat{s}+\hat{s}^{3} / 3,
$$

and we notice that we have $\partial_{\hat{\partial}} \Phi \neq 0$ for $\hat{s}= \pm \hat{s}_{0}$, and $\partial_{\hat{s}} \Phi \neq 0$ if $\hat{\sigma}=0$ or $\hat{\sigma}= \pm \hat{\sigma}_{0}$; finally on every vertex of $\partial C$, we have $\Phi \neq 0$. Therefore the boundary of $C$ does not contribute microlocally near $p_{0}$. The only contribution comes from the critical point $(\hat{\sigma}, \hat{s})=(0,0)$. With an integration by part, we can reduce the case $k=2$ to the case $k=1$, which is considered below. The change of variable $\hat{\sigma}=u^{2}, 2 d u=\hat{\sigma}^{-1 / 2} d \hat{\sigma}$, yields an integral with a holomorphic symbol and a phase given by

$$
\Phi(u, \hat{s}, \lambda)=-u^{2} \hat{s}+\hat{s}^{3} / 3-i \lambda^{-2 / 3} \hat{s} a .
$$

This phase has an isolated critical point at $(u, \hat{s})=(0,0)$, for $\lambda^{-2 / 3}=0$. We use the division theorem for symbols [BdM.] with the Jacobian ideal of (4.19), spanned by

$$
-2 u \hat{s},-u^{2}+\hat{s}^{2}-i \lambda^{-2 / 3} \frac{\partial \hat{s} a}{\partial \hat{s}} \text {. }
$$

Near zero $z=\lambda^{-2 / 3}$ is an holomorphic parameter. For $z=0$ the quotient is spanned by the four functions $l, u, \hat{s}, \hat{s}^{2}$ since the right hand side of (4.19) depends only on $u^{2}$ there are no terms in $u$. Therefore we reduce the study of (4.18) to the case where $f$ is a linear combination of the functions $1, s, s^{2}$ with coefficients which are analytic with respect to $z=\lambda^{-1 / 3}$.

Finally the right hand side of (4.19) is holomorphic with respect to the zero order term of the Taylor expansion of $a$. Deriving with respect to this parameter we reduce the study of (4.18) to the case where $f$ is constant. Then we write

$$
\int_{1-\sigma_{0}, \sigma_{0} l} e^{-i \lambda \sigma s}(\sigma+i 0)^{-1 / 2} d \sigma=\text { Constant }\left(\lambda s_{+}\right)^{-1 / 2}+e^{-i \lambda \sigma_{0} s} T^{+}(\lambda s)-e^{i \lambda \sigma_{0} s} T^{-}(\lambda s) .
$$

Here, $T^{+}(\lambda s)$ is given by:

$$
T^{+}(\lambda s)=\int_{0}^{\infty}\left(\lambda+\sigma_{0}\right)^{-1 / 2} e^{-i \lambda x s} d x
$$


which is holomorphic in the sector $-\pi-\varepsilon \leqq \operatorname{Arg} s \leqq \varepsilon, s \neq 0$ and which satisfies in this region the estimate

$$
\left|T^{+}(\lambda s)\right| \leqq \text { Constant }\left(1+|\lambda s|^{-1 / 2}\right) .
$$

Therefore, a contour deformation with respect to $s$ in the half plane $\operatorname{Im} s<0$ shows that the contribution of $T^{+}$is microlocally zero near $p_{0}$; the same remark holds for the term $T^{-}$. Finally, it is enough to study the integral

$$
I(\lambda)=\lambda^{-1 / 2} \int_{\left[0, s_{1}\right]} e^{i \lambda\left(s^{3} / 3-i \lambda^{-2 / 3} s a\right)} s^{-1 / 2} d s .
$$

In the right hand side, we replace $a$ by $a_{0}+a_{1} s$ using a change of variable which leaves the origin unchanged and which is analytic with respect to $\lambda^{-2 / 3}$; then we replace $s_{1}$ by $+\infty$. We remark that:

$$
\int_{0}^{\infty} e^{i z \xi+i \xi^{3} / 3} \xi^{-1 / 2} d \xi=\alpha \operatorname{Ai}\left(2^{-2 / 3} e^{-2 i \pi / 3} z\right) \operatorname{Ai}\left(2^{-2 / 3} z\right)
$$

with $\alpha=3^{-5 / 6} e^{i \pi / 12} \Gamma(1 / 6)(\mathrm{Ai}(0))^{-2}$, because the two sides of (4.20) satisfy the differential equation:

$$
-f^{\prime \prime \prime}+z f^{\prime}+f / 2=0,
$$

whose solutions are products of Airy functions and the identification is completed by noticing that the left hand side of $(4.20)$ is bounded for $\varepsilon<\operatorname{Arg} z<\pi-\varepsilon$. Therefore, up to a function with exponential decay in $\lambda, I(\lambda)$ is given by the series:

$$
\begin{aligned}
S(\lambda)= & \lambda^{-2 / 3} \sum_{0 \leqq k \leqq \infty}\left(\left(a_{1} \lambda^{-1 / 3}\right)^{k} / k !\right) \cdot\left(-i \partial_{z}\right)^{2 k}\left[\alpha \operatorname{Ai}\left(2^{-2 / 3} e^{-2 i \pi / 3} z\right)\right. \\
& \left.\times \operatorname{Ai}\left(2^{-2 / 3} z\right)\right]\left.\right|_{z}=-i a_{0} .
\end{aligned}
$$

The series converges because the derivatives of order $2 k$ are bounded by $C^{k}(2 k / 3)$ ! $\leqq(2 k / 3) ! \cdot(k !) \leqq e^{k / 3} k^{-k / 3}$.

It remains to show that $\mathbb{T}^{k} f_{2}$ is microlocally Gevrey 3 with weight $m^{\prime}, m^{\prime}\left(p_{0}\right)$ $>\operatorname{Re} \psi\left(p_{0}\right)$. The analytic singular support of $f$ is, by hypothesis, contained in $\{t=0, x=y\} \cup\{\Lambda \cap(t>0)\}$, we deduce from (4.7) and (4.8) that we can assume that analytic spectrum of $f_{2}$ is arbitrarily close to $p_{0}$. The result is then a consequence of the following lemma applied to the distribution kernel $\mathbb{T}^{j}(t-s, x, y) \cdot \delta_{z=w}$.

Lemma 4.2. Let $k(x, y)$ be a distribution kernel with the following properties:

(i) The analytic singular support $S S(k)$ satisfies the relation:

$$
\{S S(k) \cap \xi=0\} \cup\{S S(k) \cap \eta=0\} \subset\{\xi=\eta=0\} .
$$

(ii) For any $\varepsilon>0$ there exists a neighborhood $V$ of $\left(x_{0}, \xi_{0}\right)$ such that the relations $(x, \xi, y, \eta) \in S S(k)$ and $(y,-\eta) \in V$ imply

$$
|(x, \xi)-(y,-\eta)| \leqq \varepsilon .
$$

Then for any $\alpha>0$, there exists a neighborhood $W$ of $\left(x_{0}, \xi_{0}\right)$ such that for any function with analytic singular support containted in $W$, and which is microlocally 
Gevrey 3 with weight $m_{0}|\eta|^{1 / 3}$ on $W$, the function:

$$
g(x)=\int k(x, y) f(y) d y
$$

is microlocally Gevrey 3 at $\left(x_{0}, \xi_{0}\right)$ with weight $m_{0}(1-\alpha)|\xi|^{1 / 3}$.

Proof. We just sketch the proof. Using (4.22), we can assume that $k(x, y)$ is the boundary value of a function $h$ which is holomorphic in the region:

$$
(\operatorname{Im} x-\operatorname{Im} y) \cdot \xi_{0}>\delta_{0}[|\operatorname{Im} x|+|\operatorname{Im} y|] ;|\operatorname{Im} x|,|\operatorname{Im} y|<\delta_{1},
$$

with $\delta_{0}$ as small as we wish and, replacing $k$ by $\Delta^{-N} k$, we may assume that $k$ is bounded. Then we have:

$$
\int e^{-\lambda(z-x)^{2} / 2} k(x, y) d x=F(z, y, \lambda),
$$

where $F$ is a holomorphic function in $z$ and $y$ which satisfies the estimate:

$$
|F(z, y, \lambda)| \leqq e^{\lambda(\operatorname{lm} z-\theta \operatorname{Im} y)^{2} / 2},
$$

provided $z$ is close to $x_{0}-i \xi_{0}, \operatorname{Re} y$ is close to $x_{0}, \theta<1$ close to 1 and $\operatorname{Im} y \cdot \xi_{0}(1-\theta)$ $\leqq-\delta_{0}|\operatorname{Im} y|,|\operatorname{Im} y| \leqq \delta_{0} / 2$.

Then the ordinary F.B.I. transform of $g$ is given by

$$
\iint e^{-\lambda(z-x)^{2} / 2} k(x, y) f(y) d y d x=\int F(z, y, \lambda) f(y) d y .
$$

The demonstration is completed by expressing $f$ using the inversion formula for the F.B.I. transform, and then deforming the contour in $y$. $f \in I_{A, \psi}^{3}$

The next proposition describes the action of the operators $\mathbb{T}^{k}$ on the symbol of Proposition 4.2. Let $f=f_{1}+f_{2} \in I_{A, \psi}^{3}$ with $f_{2}$ microlocally Gevrey 3 with weight larger than $\operatorname{Re} \psi\left(p_{0}\right)$ and with $f_{1}$ having symbol a

$$
f_{1}(t, x, z)=\int e^{i \varphi(t, x, z, \theta)-l(t, x, z, \theta)} a(t, x, z, \theta) d \theta
$$

and let $b^{k}(t, x, z, \theta)$ be the symbol of $\mathrm{T}^{k} f$

$$
\left(T^{k} f\right)_{1}(t, x, z)=\int e^{i \varphi(t, x, z, \theta)-l(t, x, z, \theta)} b^{k}(t, x, z, \theta) d \theta .
$$

Then, the symbol $b^{k}$ can be computed from the symbol a according to the formula

$$
b^{k}={\widehat{T^{k}}}^{a} a=\Theta^{2 / 3-4 k / 3} \sum_{n=0}^{\infty} \Theta^{-n / 3} Q_{n}^{k}(a),
$$

where

$$
\left.Q_{n}^{k}\left(t, x, z, \theta /|\theta|, D_{t}, D_{x}\right)=\sum_{|\beta| \leqq n} Q_{n, \beta}^{k}(t, x, z, \theta /|\theta|)\right) \partial_{t, x}^{\beta}
$$

is a differential operator of degree less than or equal to $n$, and, the coefficient $Q_{n, \beta}^{k}$ are holomorphic functions satisfying the estimates:

$$
\left|Q_{n, \beta}^{k}\right| \leqq C^{n}(n-|\beta|) ! .
$$


Furthermore $Q_{0}^{1}$ is identically equal to zero and for the principal symbol denoted by $\sigma$, we have the relations

$$
\sigma\left(\mathbb{T}^{1} a\right)=C_{1} \Theta^{-1} \varrho^{-1}\{\tau-\Theta, \sigma(a)\}, \quad \sigma\left(\mathbb{T}^{2} a\right)=C_{2} \Theta^{-2} \varrho^{-1} \sigma(a),
$$

where $C_{1}$ and $C_{2}$ are universal constants in $\mathbb{C} \backslash 0$ and where $\Theta=\Theta\left(x, x, v_{0}\right)$.

Proof. We follow the idea of the proof of the Proposition 4.1 using coordinates as in (4.13). With $\mathbb{T}^{k}$ given by (4.12), we have:

$H^{k}(x, y, \sigma, v)=2^{-k}(2 \pi)^{-n}[\Theta(x, y, v)]^{2-2 k}(2-\sigma)^{1 / 2-k}\left[\left(1+\left(\partial_{y} \cdot S\right)^{2}\right) /\left(1+(H(x, y))^{2}\right)\right]^{1 / 2}$

Let

$$
f^{k}(t, x, z, \theta, \sigma, s) \equiv e^{l\left(s, y_{c}, z, \theta\right)} Q\left(H^{k} a e^{-l(s, y, z, \theta)}\right)_{\mid y=y_{c}, v=v_{c}}
$$

the function which appears in (4.16) and we have:

$$
f^{k}=\sum_{0 \leqq m<\infty}|\theta|^{-m / 3} F_{m}\left(H^{k} a\right),
$$

where $F_{m}$ is a differential operator of degree $2 m / 3$. Since $\Theta(x, x, v)=|v|$ and $\operatorname{det}\left[\Theta_{v, v}^{\prime \prime}(x, x, v)\right]=0$, we have:

$$
F_{0}=(2 \pi)^{n-1}\left(1+O\left((t-s)^{2}\right)\right) .
$$

The coefficients $b^{k}(t, x, z, \theta)$ are obtained from the asymptotic expansion, with respect to $\Theta\left(x, x, v_{0}\right)$ of the expression

$$
\begin{aligned}
& \iint_{\operatorname{Im} \sigma>0} \exp \left\{i \Theta\left[\sigma(s-t)-\varrho^{2}(s-t)^{3} / 24+O\left((s-t)^{4}+\sigma(s-t)^{2}\right)\right]\right. \\
& \left.\quad+\Theta^{1 / 3}\left[(t-s) \varrho^{2 / 3} e^{i \pi / 6} 2^{-1 / 3} \omega+O\left((s-t)^{4}+\sigma(s-t)^{2}\right)\right]\right\} \sigma^{1 / 2-k} \tilde{f}^{k}(\sigma, s) d \sigma d s .
\end{aligned}
$$

To obtain the asymptotic expansion, it is convenient to introduce the change of scale:

$$
\sigma=\Theta^{-2 / 3} \beta, \quad(t-s)=\Theta^{-1 / 3} \gamma .
$$

The terms $O\left[(s-t)^{4}+\sigma(s-t)^{2}\right]$ of the phase then contribute only lower order terms to the symbol $f^{k}$ and we denote $f^{k}$ the new symbol which is obtained in this way. We have to compute the principal term of:

$$
\begin{aligned}
I(\Theta)= & \Theta^{2 k / 3-4 / 3} \iint_{1>0} e^{i\left[\gamma^{3} \Theta^{2} / 24+\gamma\left(-i \boldsymbol{Q}^{2 / 3} e^{i \pi / 6} 2^{-1 / 3} \omega\right)-\beta \gamma\right]} \\
& \times \beta^{1 / 2-k} \tilde{f}^{\operatorname{Im} \beta}\left(\Theta^{-2 / 3} \beta, t-\Theta^{-1 / 3} \gamma\right) d \gamma d \beta .
\end{aligned}
$$

We obtain the formal expansion

$$
\begin{gathered}
I(\Theta)=\Theta^{2 k / 3-4 / 3} \sum_{j_{1}, j_{2}} \Theta^{-\left(2 j_{1}+j_{2}\right) / 3}\left((-1)^{j_{2}} /\left(j_{1} !\right)\left(j_{2} !\right)\right. \\
\times\left(\partial_{\sigma}^{j_{1}} \partial_{s}^{j_{2}} f^{k}(\sigma, s)\right)_{\sigma=0, s=t}\left(2 \varrho^{-2 / 3}\right)^{j_{2}-j_{1}+k-1 / 2} \cdot C_{j_{1} j_{2}}^{k}\left(e^{-i \pi / 3} 2^{2 / 3} \omega\right) .
\end{gathered}
$$

Here $C_{j_{1} j_{2}}^{k}(z)$ denotes the entire function of $z$ given by:

$$
C_{j_{1} j_{2}}^{k}(z)=\int e^{i\left(\xi^{3} / 3+z \xi\right) \xi^{j_{2}}} d \xi \int_{\operatorname{Im} x>0} e^{-i x \xi} x^{1 / 2-k+j_{1}} d x .
$$


From the formula (4.27), we deduce that the $Q_{n}^{k}$ are differential operators of degree at most $n$, because $n$ is the maximum of $2 m / 3+j_{1}+j_{2}$ under the condition $2 j_{1}+j_{2}+m=n$; it is obtained for $m=j_{1}=0$ and $j_{2}=n$. The estimations (4.23) are consequences of the Proposition 4.1 which implies that $\mathbb{T}^{k}$ is a continuous mapping of the space of regular symbols with respect to $\Theta^{1 / 3}$ to itself. The fact that $Q_{0}^{1}$ is identically zero is a consequence of the formula (4.20) which implies that one has:

$$
\left.C_{0,0}^{1}(z)=\text { Constant } \cdot \operatorname{Ai}\left(e^{-2 i \pi / 3} 2^{-2 / 3} z\right) \operatorname{Ai}\left(2^{-2 / 3}\right) z\right)
$$

and with $z=2^{2 / 3} e^{-i \pi / 3} \omega$ we have:

$$
\operatorname{Ai}\left(e^{-2 i \pi / 3} 2^{-2 / 3}(z)\right)=\operatorname{Ai}(-\omega)=0 .
$$

The leading term of $\mathbf{T}^{1} a$ is obtained for $j_{1}=0$ and $j_{2}=1$

$$
C_{0,1}^{1}=\text { Constant }\left.\partial_{z}\left(\operatorname{Ai}\left(e^{-2 i \pi / 3} 2^{-2 / 3} z\right) \operatorname{Ai}\left(2^{-2 / 3} z\right)\right)\right|_{z=2^{2 / 3} e^{-i \pi / 3} \omega} .
$$

Similarly, the leading term of $\mathbf{T}^{2} a$ is obtained for $j_{1}=j_{2}=0$ and it is also of the form:

$$
C_{0,0}^{2}=\text { Constant }\left.\partial_{z}\left(\operatorname{Ai}\left(e^{-2 i \pi / 3} 2^{-2 / 3} z\right) \operatorname{Ai}\left(2^{-2 / 3} z\right)\right)\right|_{z=2^{2 / 3} e^{-i \pi / 3} \omega} .
$$

The relations (4.24) follow form (4.25), (4.26), and (4.27).

\section{Proof that $k^{+}(t, x, y) \in I_{A, \psi}^{3}$}

This section is devoted to proving that $k^{+} \in I_{A, \psi}^{3}$ in $t>0$ with $A, \psi$ defined in Eqs. (4.9), (4.10). The proof has two major steps, first the result is obtained for small $t$ (Proposition 5.2), using a parametrix construction of Lebeau [L.2]. The result is extended to all times using the methods of second microlocalization as in $[\mathrm{L} .2, \S 4],[\mathrm{L} .2, \S 5]$, [S.K.K.] and [S.1] and a transport equation involving the operators $\mathbb{T}^{k}$ of the last section.

The parametrix is constructed using families $f(t, x, \lambda), \lambda \geqq \lambda_{0}$ of functions holomorphic with respect to $t, x$.

Suppose $f: \omega \times\left(\lambda_{0}, \infty\right), \omega$ a neighborhood in $\mathbb{C}_{t} \times \mathbb{C}_{x}$ of $\left(t_{0}, x_{0}\right)$, is holomorphic in $t, x$ and satisfies:

$$
\begin{gathered}
(\exists C>0) \quad|f(t, x, \lambda)| \leqq e^{C \lambda}, \quad t, x, \lambda \in \omega \times\left[\lambda_{0}, \infty\right], \\
\left(\exists N_{0}\right)(\forall \alpha)\left(\exists C_{\alpha}\right) \quad\left|\partial_{t, x}^{\alpha} f(t, x, \lambda)\right| \leqq C_{\alpha} \lambda^{N_{0}+|\alpha|} \quad t, x, \lambda \in(\omega \cap \mathbb{R} \times \Omega) \times\left[\lambda_{0}, \infty\right] .
\end{gathered}
$$

(The exponential versus polynomial growth in these relations comes from the fact that in (5.1) $x$ and $t$ are complex while in (5.2) they are real.)

Definitions 5.1. The asymptotic singular spectrum of $f(t, x, \lambda)$, denoted by $S S_{\partial} f$ is the closed set of $T^{*}\left(\mathbb{R}_{t} \times \partial \Omega\right) \cup T^{*}\left(\mathbb{R}_{t} \times \Omega\right)$ defined as follows: An interior point $p_{0} \in T^{*}\left(\mathbb{R}_{t} \times \Omega\right)$ does not belongs to $S S_{\hat{o}}$, there exists a F.B.I. transform:

$$
f \rightarrow \int e^{i \lambda g(z, t, x)} f(t, x, \lambda) d x,
$$


with value in the space $H_{\varphi}$, related to the canonical transform:

$$
\chi:\left(T^{*}\left(\mathbb{R}_{t} \times \Omega\right), p_{0}\right) \rightarrow\left(\Lambda_{\varphi}, z_{0}\right),
$$

for which $f$ satisfies in some complex neighborhood $\omega_{0}$ of $z_{0}$, and with some strictly positive constant $C_{0}$, the following estimate:

$$
\forall z \in \omega_{0}, \forall \lambda \geqq \lambda_{0}, \quad\left|\int e^{i \lambda g(z, t, x)} f(t, x, \lambda) d t d x\right| \leqq C e^{\lambda\left(\varphi(z)-C_{0}\right)} .
$$

$A$ boundary point $q_{0} \in T^{*}\left(\mathbb{R}_{t} \times \partial \Omega\right)$ does not belong to $S S_{\partial} f$ if in a system of $C^{\omega}$ local coordinates $x^{\prime}, l$ with $\Omega=\{l>0\}$ there is a tangential F.B.I.

$$
f \rightarrow \int e^{i \lambda g\left(z^{\prime}, t^{\prime}, x^{\prime}\right)} f\left(t, x^{\prime}, l, \lambda\right) d x^{\prime} d t,
$$

with values in $H_{\varphi^{\prime}}$ related to the canonical transformation

$$
\chi^{\prime}:\left(T^{*}\left(\mathbb{R}_{\mathrm{t}} \times \partial \Omega\right), q_{0}\right) \rightarrow\left(\Lambda_{\varphi^{\prime}}, z_{0}^{\prime}\right)
$$

and $C, C_{0}>0$ such that for all $z^{\prime} \in \omega_{0} C \mathbb{C}^{n-1}$ a complex neighborhood of $z_{0}^{\prime}, 0 \leqq l<l_{0}$, and $\lambda>\lambda_{0}$

$$
\left|\int e^{i \lambda g\left(z^{\prime}, t, x^{\prime}\right)} f\left(t, x^{\prime}, l, \lambda\right) d t d x^{\prime}\right| \leqq C e^{\lambda\left(\varphi^{\prime}\left(z^{\prime}\right)-C_{0}\right)} .
$$

We say that $f$ is an asymptotic solution if there exists constants $C, C_{0}>0$ so that

$$
\left|\left(\partial_{t}^{2}-\Delta_{x}\right) f(t, x, \omega)\right| \leqq C e^{-C_{0} \lambda}
$$

for all $t, x, \in \omega, \lambda \geqq \lambda_{0}$.

We denote by $q_{0} \in T^{*}\left(\mathbb{R}_{t} \times \partial \Omega\right)$ a point of the form $q_{0}=\left(t_{0}, x_{0},-\tau_{0},-\xi_{0}^{\prime}\right)$ with $\tau_{0}=\left|\xi_{0}^{\prime}\right|$ and, for $q \in T^{*}\left(\mathbb{R}_{t} \times \partial \Omega\right)$ near $q_{0}$, we define the incoming flow $F^{-}(q)$. The definition is different in the elliptic $\left(|\tau|<\left|\xi^{\prime}\right|\right)$, hyperbolic $\left(|\tau|>\left|\xi^{\prime}\right|\right)$, and glancing $\left(|\tau|=\left|\xi^{\prime}\right|\right)$ sets.

$$
\text { If } q \text { is elliptic, then } F^{-}(q)=\{q\} \text {. }
$$

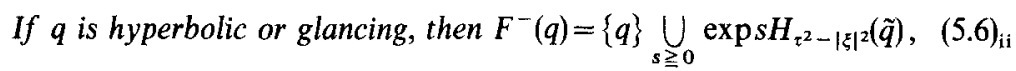
where $\tilde{q}=\left(t_{0}, x_{0}, \tau, \xi^{\prime}, \xi_{n}\right)$ is the characteristic point projecting to $q$ such that the bicharacteristic $\exp s H_{\tau^{2}-|\xi|^{2}}(q)$ lies over $\Omega$ for $s>0$. Notice that on bicharacteristics $d t / d s \simeq-2|\xi|<0$ so $s>0$ corresponds to the past.

Definition 5.2. We say that $f(t, x, \lambda)$ is an incoming asymptotic solution if it is asymptotic solution such that $f_{0}\left(t, x^{\prime}, \lambda\right) \equiv f\left(t, x^{\prime}, 0, \lambda\right)$ satisfies

$$
\begin{aligned}
& S S\left(f_{0}\right) \subset \omega_{0}, \text { a small neighborhood of } q_{0} \text { in } T^{*}\left(\mathbb{R}_{t} \times \partial \Omega\right) \\
& S S_{\partial}(f) \subset F^{-}\left[S S\left(f_{0}\right) \cup\left(\bigcup_{s>0} \operatorname{exps} H_{\tau^{2}-\left|\xi^{\prime}\right|^{2}}\left(S S\left(f_{0}\right) \cap G\right)\right)\right],
\end{aligned}
$$

where $G \equiv$ the glancing set.

In Fig. 1, we sketch the projection on $(t, x)$ of the singularities which correspond to the relation (5.8) and which come from the points $\left(t_{0}, x_{0}, \tau, \xi\right)$, for $(\tau, \xi)$ near 


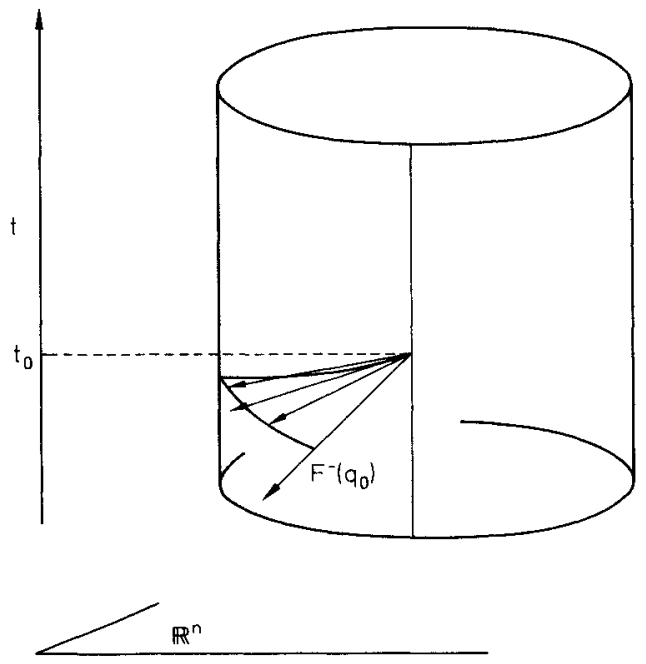

Fig. 1

$\left(\tau_{0}, \xi_{0}\right) \in G$. This set is the union of a part of the wave cone coming from $\left(t_{0}, x_{0}\right)$ and of the rays which are glancing along the backward geodesic through $x_{0}$.

One can find (cf. [S2] a system of coordinates and a positive function $a(x)$ such that, near $x_{0}$, one has:

$$
a^{-1 \circ} \Delta_{x} \circ a=\partial_{l}^{2}+B\left(x^{\prime}, l, D_{x}\right),
$$

where $l$ denotes the variable normal to $\partial \Omega$ and where $B$ is a second order elliptic operator with

$$
\sigma(B)\left(x^{\prime}, 0, \xi^{\prime}\right)=-\left|\xi^{\prime}\right|^{2} .
$$

Therefore, after a conjugation with the function $a$, the wave operator becomes:

$$
P=a^{-1}\left(\Delta_{x}-\partial_{t}^{2}\right) a=\left(\partial_{l}^{2}\right)+\left(\left(B\left(x^{\prime}, l, D_{x}\right)-\left(\partial_{t}^{2}\right)\right.\right.
$$

with symbol $p=-\xi_{n}^{2}+\left(B\left(x^{\prime}, l, \xi^{\prime}\right)+\tau^{2}\right)$.

After this reduction, we make a tangential F.B.I. transform

$$
T f(z, l, \lambda) \equiv \int e^{i \lambda g_{0}\left(z, t, x^{\prime}\right)} f\left(t, x^{\prime}, l, \lambda\right) d x^{\prime} d t
$$

for $z$ near $z_{0}$ in $\mathbb{C}^{n}$. The transform is related to a canonical transform

$$
\chi_{0}: T^{*}\left(\mathbb{R}_{t} \times \partial \Omega\right) \rightarrow\left(\mathbb{C}^{n},-2 i \bar{\partial} \partial \varphi_{0}\right), \varphi_{0} \equiv 1 / 2(\operatorname{Im} z)^{2}, \chi_{0}(G)=\left\{z \mid \operatorname{Im} z_{1}=0\right\} .
$$

As in [L.2], the operator $P$ is transformed to

$$
P=\partial_{l}^{2}+\lambda^{2} R(l, z, \xi, \lambda),
$$

where $R$ is a classical $C^{\omega}$ symbol of degree zero,

$$
R \sim \sum_{j \geqq 0} R_{j}(l, z, \lambda) \lambda^{-j}
$$


The characteristic variety, $0=\sigma(P)=R_{0}-T^{2}(\tau$ dual to $l$ ), is given by

$$
l=-q\left(z, \xi, \tau^{2}\right)=-\xi_{1}^{\prime}+e(z, \xi) I^{2}+O\left(T^{4}\right)
$$

with $e(0,0)>0$.

Incoming asymptotic solutions to the wave equation are constructed in two steps, following [L.2, Prop. 3.3 and 4.2.3.]. First incoming asymptotic solutions of $P=0$ are constructed, then an inverse tangential F.B.I. is applied. The next result lifted from [L.2] accomplishes the first step. Note that $T^{*}\left(\mathbb{R}_{t} \times \partial \Omega\right)$ and $\mathbb{C}_{z}^{n}$ are identified using $\chi_{0}$.

Proposition 5.1. There exists a linear operator $K$ which maps the set of function $f_{0}$ satisfying the relations $\left|f_{0}(z, \lambda)\right| \leqq e^{\lambda \varphi_{0}(z)}$, and, for $|z|>\delta / 2, \delta>0$ small $\left|f_{0}(z, \lambda)\right|$ $\leqq e^{\lambda\left(\varphi_{0}(z)-C\right)}, C>0$. To the set or incoming asymptotic solutions of $P=0$ with the following properties:

$$
(K f)(0, z, \lambda)=f_{0}(z, \lambda)+O\left(e^{-C \lambda}\right),
$$

and

$$
\partial_{l}(K f)(0, z, \lambda)=(\lambda / 2 \pi)^{n-1} \int d y^{\prime} \wedge d \xi^{\prime}
$$

$$
\times \int_{-2 \delta / 3}^{2 \delta / 3} e^{i \lambda\left(z^{\prime}-y^{\prime}\right) \cdot \xi^{\prime}-i \lambda^{1 / 3}\left(H\left(z, \xi^{\prime}\right)-H\left(y, \xi^{\prime}\right)\right)} k\left(z_{1}, z^{\prime}, y_{1}, \xi^{\prime}, \lambda\right) f(y, \lambda) d y_{1}+O\left(e^{-c \lambda}\right) .
$$

for $z$ near $\left(z_{1}^{0}, 0\right), z_{1}^{0} \in \mathbb{R}, z_{1}^{0} \geqq r_{0} \geqq 2 \delta$. The holomorphic function $H\left(z, \xi^{\prime}\right)$ satisfies the relation

$$
\partial_{z_{1}} H\left(z, \xi^{\prime}\right)=\omega e^{-i \pi / 3}\left(e\left(z, \xi_{1}=0, \xi^{\prime}\right)\right)^{1 / 3},
$$

where $e(z, \xi)$ is given by (5.14). $k$ is an analytic symbol in a small neighborhood $W$ (independent of $\delta$ and $r_{0}$ ) of $z_{1}=0, y_{1}=0, z^{\prime}=0, \xi^{\prime}=0$. In addition, there exists $a$ family $k^{j}$ of holomorphic functions defined in $W$ which satisfy the following estimate:

$$
\begin{gathered}
\left|k^{j}\right| \leqq A B^{j} j !,\left.\quad k_{0}\right|_{x_{1}=y_{1}} \cong i, \\
\left|k\left(z_{1}, z^{\prime}, y_{1}, \xi^{\prime}, \lambda\right)-\sum_{0 \leqq 1 \leqq j} k^{l}\left(z_{1}, z^{\prime}, y_{1}, \xi^{\prime}\right) \lambda^{1-l / 3}\right| \leqq A\left(r_{0}\right) B\left(r_{0}\right)^{j^{j} ! \lambda^{1-j / 3}} .
\end{gathered}
$$

Remark 5.1. Near $q_{0}=\left(t_{0}, x_{0}^{t},-\tau_{0},-\xi_{0}^{\prime}\right)$, the incoming asymptotic solutions propagate singularities in the direction of negative times; they coincide with the outgoing solutions in the sense of [L.2]. Indeed, with $P$ given by (5.13), they propagate the singularities to the right along the bicharacteristic. We have used the minus sign in front of $\tau_{0}$ to recover the definition given in [L.2].

There exists an analytic, elliptic, classical symbol, $\sigma_{0}$, of degree zero such that the F.B.I.

$$
f \rightarrow T^{-1} f=\lambda^{n} \int e^{-i \lambda g_{0}\left(z, t, x^{\prime}\right)} \sigma_{0}(t, x, z, \lambda) f(l, z, \lambda) d z
$$

is a formal inverse of the F.B.I. defined by (5.12).

For $\left(z,-2 i \partial_{z} \varphi_{0}(z)\right) \in \chi\left(T_{(t, x)}^{*}\left(\mathbb{R}_{1} \times \partial \Omega\right)\right)$, the function $z \rightarrow \operatorname{Im} g_{0}\left(z, t, x^{\prime}\right)+\varphi_{0}(z)$ has its critical points transversally non degenerate, and the critical value is zero with signature $\left(n^{+}, 0^{-}\right)$. Then if $f(l, z, \lambda)$ is an incoming asymptotic solution with 
$S S_{\partial} f(0, z, \lambda) \subset\left|z-z_{0}\right|<\varepsilon_{0} / 4$, the function given by

$$
f(t, x, \lambda)=a(x) \lambda^{n} \int_{z \in \Sigma_{0}} e^{-i \lambda g_{0}\left(z, t, x^{\prime}\right)} \sigma_{0} f(l, z, \lambda) d z
$$

with

$$
\Sigma_{0} \equiv\left\{z\left|\left(z,-2 i \partial_{z} \varphi_{0}(z)\right)=\chi(t, x ; \tau, \xi),\right|(\tau, \xi)-\left(-\tau_{0},-\xi_{0}\right) \mid \leqq \varepsilon_{0}\right\}
$$

is an incoming asymptotic solution of the wave equation, for

$$
\left|(t, x)-\left(t_{0}, x_{0}\right)\right| \leqq \varepsilon_{1}, \quad \varepsilon_{1} \ll \varepsilon .
$$

Let $q_{0}^{(a)} \equiv\left(t_{0}, x_{0}^{\prime} ; \tau_{0}, \xi_{0}\right)$ be the antipodal of $q_{0}$ and let

$$
f \rightarrow T_{1} f \equiv \int e^{i \lambda g_{1}\left(z, t, x^{\prime}\right)} f\left(t, x^{\prime}, \lambda\right) d x^{\prime} d t
$$

be a F.B.I. transform related to the canonical transform

$$
\chi_{1}:\left(T^{*}\left(\mathbb{R}_{t} \times \partial \Omega\right) ; q_{0}^{(a)}\right) \rightarrow\left(\mathbb{C}^{n},(2 / i) \partial \partial \varphi_{1}\right) .
$$

For $z$ near $z_{1}$ (considered as a parameter), and $(t, x)$ near $\left(t_{0}, x_{0}\right)$ we denote by $f(t, x ; z, \lambda)$ the incoming asymptotic solution, described above, which satisfies the boundary condition:

$$
f\left(t, 0, x^{\prime} ; z, \lambda\right)=a\left(0, x^{\prime}\right) e^{i \lambda g_{1}\left(z, t, x^{\prime}\right)-\lambda \varphi_{1}(z)}+O\left(e^{-C \lambda}\right) .
$$

For $\left|z-z_{1}\right| \ll \varepsilon_{2}, f$ decays exponentially with respect to $\lambda$ for $t \geqq t_{0}+\varepsilon_{2}$. The kernel $K^{+}(t-s, x, y)$ from (2.9) has its support contained in the cone $|x-y| \leqq|t-s|$, therefore, if $\left|t_{0}-s\right|$ and $\left|x_{0}-y\right|$ are small enough, we have

$$
O\left(e^{-C \lambda}\right)=\int_{\Omega} d x \int_{-\infty}^{t_{0}+\varepsilon_{2}} d t\left[\left(\partial_{t}^{2}-\Delta_{x}\right) f(t, x, z, \lambda)\right] K^{+}(t-s, x, y) .
$$

Integrating by parts in the right hand side yields

$$
\int_{\partial \Omega} d x^{\prime} \int d t a\left(0, x^{\prime}\right) e^{i \lambda g_{1}\left(z, t, x^{\prime}\right)-\lambda \varphi_{1}(z)} k^{+}\left(t-s, x^{\prime}, y^{\prime}\right)=\partial_{n} f\left(s, y^{\prime}, z, \lambda\right)+O\left(e^{-c \lambda}\right) .
$$

This duality argument leads to

Lemma 5.1. The analytic singular spectrum of $k^{+}\left(t, x^{\prime}, y^{\prime}\right)$ is, for $t>0$, contained in A.

Proof. Since $k^{+}$is real, it is enough to prove the relation: $\left\{S S\left(k^{+} \cap t>0 \cap \tau>0\right\}\right.$ $\subset A^{+}$. For $t$ small, one applies to both sides of the formula (5.18), a F.B.I. transform with respect to the variables $\left(s, y^{\prime}\right)$, and uses the result of Sjöstrand [S.2] describing the propagation of analytic singularities.

Proposition 5.2. For $t>0$ small enough, the kernel $k^{+}$belongs to the class $I_{\Lambda, \psi}^{3}$.

Proof. It suffices to consider $A^{+}$. For $s<t_{0}$ and $\left|z-z_{1}\right| \ll t_{0}-s$, we deduce from the formulas (5.15) and (5.17) that for $\left(w,-2 i \partial_{w} \varphi_{0}(w)\right)$ near $\chi_{0}\left(\exp \left(\left(t_{0}-s\right) H_{\tau-\left|\xi^{\prime}\right|}\left(q_{0}\right)\right)\right.$, 
one has

$$
\begin{gathered}
\int\left(e^{i \lambda g_{0}\left(w, s, y^{\prime}\right)}\left(a\left(0, y^{\prime}\right)\right)^{-1} \partial_{n} f\left(s, y^{\prime}, z, \lambda\right) d s d y^{\prime}\right. \\
=(\lambda / 2 \pi)^{n-1} \int d u^{\prime} \wedge d \xi^{\prime} \int d u_{1} e^{i \lambda\left(w^{\prime}-u^{\prime}\right) \xi^{\prime}-i \lambda^{1 / 3}\left[H\left(w, \xi^{\prime}\right)-H\left(u, \xi^{\prime}\right)\right]} \\
\times k\left(w_{1}, w^{\prime}, u_{1}, \xi^{\prime}, \lambda\right) \int e^{i \lambda g_{0}\left(u, t, x^{\prime}\right)+i \lambda g_{1}\left(z, t, x^{\prime}\right)-\lambda \varphi_{1}(z)} d t d x^{\prime} .
\end{gathered}
$$

The phase $g_{0}+g_{1}$ which appears in the right hand side defines an F.B.I. transform related to the canonical transform.

$$
\chi_{1} \otimes \chi_{0}: T^{*}\left(\mathbb{R}_{t} \times \partial \Omega_{x^{\prime}}\right) \times T^{*}\left(\mathbb{R}_{t} \times \partial \Omega_{y^{\prime}}\right) \rightarrow \Lambda_{\varphi_{1}} \times \Lambda_{\varphi_{0}} \subset T^{*}\left(\mathbb{C}_{z}^{n}\right) \times T^{*}\left(\mathbb{C}_{z}^{n}\right) .
$$

Therefore we can use the results of the Sect. 3. First notice that the phase

$$
\left(u^{\prime}, \xi^{\prime} ; u_{1}, t, x^{\prime}\right) \rightarrow\left(w^{\prime}-u^{\prime}\right) \cdot \xi^{\prime}+g_{0}\left(u, t, x^{\prime}\right)+g_{1}\left(z, t, x^{\prime}\right)
$$

has, for each $(z, w)$, a non degenerate critical point. The critical value $\Phi(z, w)$ is the p.s.h. function which is canonically related to the Lagrangian manifold:

$$
\chi_{1} \otimes \chi_{0}\left[\left\{\left(t, x^{\prime}, \tau, \xi^{\prime}\right)=\exp l H_{\tau-\left|\xi^{\prime}\right|}\left(s, y^{\prime},-\sigma,-\eta^{\prime}\right) ; \tau=\left|\xi^{\prime}\right|, \sigma=-\left|\eta^{\prime}\right|\right\}\right] .
$$

This follows from the fact that the phase

$$
\left(t, x^{\prime}\right) \rightarrow g_{0}\left(u, t, x^{\prime}\right)+g_{1}\left(z, t, x^{\prime}\right)
$$

has a non degenerate critical point, and the critical value $G(u, z)$ satisfies

$$
\left\{\left(z, u ; G_{z}^{\prime}, G_{u}^{\prime}\right)\right\}=\left(\chi_{1} \otimes \chi_{0}\right)\left(A_{l d}\right) \text {. }
$$

Here $\Lambda_{I d}$ is the Lagrangian manifold $t=s, x^{\prime}=y^{\prime}, \tau+\sigma=0, \xi^{\prime}+\eta^{\prime}=0$. This Lagrangian intersects transversally the manifold $\tau=\left|\xi^{\prime}\right|$.

The weight function $\psi$, homogeneous of degree $1 / 3$, is computed from the right hand side of (5.19) as is done in Theorem 1 of [L.2]. That is, $\psi$ is obtained upon integrating the 1 -form $e^{i \pi / 6} 2^{-1 / 3} \omega J$ along the bicharacteristics following $G$. Here $J$ satisfies:

$$
\left\langle J, H_{|\xi|-\tau^{2}}\right\rangle=\left\{\left\{x_{n}-S\left(x^{\prime}\right), \xi^{2}-\tau^{2}\right\}, \xi^{2}-\tau^{2}\right\}^{2 / 3} /\left\{\left\{x_{n}-S\left(x^{\prime}\right), \xi^{2}-\tau^{2}\right\}, x_{n}-S\left(x^{\prime}\right)\right\}^{1 / 3} .
$$

That this yields the $\psi$ defined in (4.10) is verified by performing the above computation in coordinates satisfying (4.13). This completes the proof Proposition 5.1.

To extend this result to all positive times, we will use the notion of partial Gevrey 3 regularity on a $C^{\infty}$ involutive submanifold $V C T^{*} M \backslash 0$. Toward this end suppose that $V \subset T^{*} \mathbb{R}^{N} \backslash 0$ is an involutive manifold with its natural Hamilton foliation. In our example, $V$ will be

$$
V^{+}=\left[(t, x, y, \tau, \xi, \eta) \in T^{*}\left(\mathbb{R}_{t} \times \partial \Omega \times \partial \Omega\right) \backslash 0|\tau=| \xi \mid\right\},
$$

and the leaves of the foliation are the integral curves of $H_{\tau-|\xi|}$. Let $\widetilde{V}$ be the manifold obtained upon complexifying the leaves. Suppose $m=\tilde{V} \rightarrow R$ is pluriharmonic on the leaves of $\widetilde{V}$ and homogeneous of degree $1 / 3$. In our example, $m$ will 
be the function $\operatorname{Re} \psi: \tilde{V}^{+} \rightarrow R$ defined by (4.10). Note that $V^{+} \supset \Lambda^{+}$and the restriction of $\psi$ to $A$ is the weight defining $I_{A, \psi}^{3}$.

Definition 5.3. Suppose that $V$ and $m$ are as above and that $T$ is an F.B.I.transform associated with $M, \chi, \varphi, d x, p_{0} \in T^{*} M$ and let $\varphi_{V}$ be the p.s.h. defined by $\chi(\widetilde{V}) c f .[\mathrm{L} .1$, $\S$ II.5]. Then $f \in D(M)$ is partially Gevrey 3 on $V$ with weight $m$ at $p_{0}$ if for $z$ in a neighborhood of $z_{0}=\pi \circ \chi\left(p_{0}\right)$ and $\lambda>\lambda_{0}, T_{\chi} f$ satisfies:

$$
(\forall \varepsilon>0)\left(\exists C_{\varepsilon}\right) \quad|T f(z, \lambda)| \leqq C_{\varepsilon} e^{\lambda \varphi_{V}(z)-\lambda^{1 / 3} m(z)+\varepsilon \lambda^{1 / 3}},
$$

where $m$ is defined by $\hat{m}^{\circ} \circ \pi_{z} \circ \chi^{\mathbb{C}}=m$.

We write $f$ is V.m.p. $G^{3}$ at $p_{0}$. To show that $k \in I_{A, \psi}^{3}$ for all $t>0$, we must show that $k$ is in this space microlocally at all points

$$
p\left(t ; y^{\prime}, \eta^{\prime}\right) \equiv\left(t, x^{\prime}(t), y^{\prime} ; \tau, \xi^{\prime}(t), \eta^{\prime}\right),\left(x^{\prime}(t), \xi^{\prime}(t)\right)=\exp -t H_{\left|\xi^{\prime}\right|}\left(y^{\prime},-\eta^{\prime}\right),
$$

where $y^{\prime}, \eta^{\prime} \in T^{*}(\partial \Omega)$ and $t>0$ is arbitrary. It is sufficient to show that if $k \in I_{A, \psi}^{3}$ microlocally at all such points for $0<t<t_{0}$ then the same is true for $t=t_{0}$. The first step is partial Gevrey 3 regularity.

Lemma 5.2. If for all $0<t<t_{0}$, and $y^{\prime}, \eta^{\prime} \in T^{*}(\partial \Omega)$, we have $k \in I_{A^{+}, w}^{3}$ at $p\left(t ; y^{\prime}, \eta^{\prime}\right)$ then for all $\left(y^{\prime}, \eta^{\prime}\right) \in T^{*}(\partial \Omega), k$ is partially Gevrey 3 on $V$ with weight $\operatorname{Re} \psi$ at $p\left(t_{0}, y^{\prime}, \eta^{\prime}\right)$.

Proof. Choose $\theta \in G^{3 / 2}(\mathbb{R})$ with $\theta=0$ on $[-\infty,-1]$ and $\theta=1$ on $[1, \infty]$. For $q_{0}^{a}$ $\equiv\left(t_{0}, x_{0}^{\prime} ; \tau_{0}, \xi_{0}^{\prime}\right) \in T^{*}\left(\mathbb{R}_{t} \times \partial \Omega\right)$ with $\left(x_{0}^{\prime}, \xi_{0}^{\prime}\right)=\exp -t H_{\left|\xi_{0}\right|}\left(y_{0}^{\prime},-\eta_{0}^{\prime}\right)$ let $f(t, x, z, \lambda)$ be the incoming asymptotic solution (5.16) with boundary values given by (5.17). For $\delta$ small and $0<\varepsilon \ll \delta$ define $w_{\varepsilon}\left(t, x, y^{\prime}\right), t, x, y^{\prime} \in \mathbb{R}_{t} \times \mathbb{R}^{n} \times \partial \Omega$, by

$$
\left(\partial_{t}^{2}-\Delta_{x}\right) w_{\varepsilon}=\theta\left(\left(t-t_{0}+\delta\right) / \varepsilon\right) k^{+}\left(t, x^{\prime}, y^{\prime}\right) \otimes \delta_{\partial \Omega}
$$

$w_{\varepsilon}=0$ for $t<t_{0}-\delta-\varepsilon$.

The asymptotic incoming solution $f(t, x, z, \lambda)$ decays exponentially in $\lambda$ away from the projection on $\mathbb{R}_{t} \times \mathbb{R}_{x}^{n}$ of a small neighborhood of $F^{-}\left[\left(q_{0}\right)\right.$

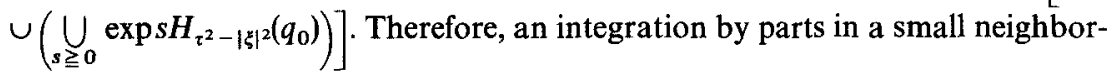
hood of $x_{0}$ in $\Omega$, yields (cf. formula (5.18)):

$$
\begin{aligned}
& \left.\iint d x d t a\left(0, x^{\prime}\right) e^{i \lambda g_{1}\left(z, t, x^{\prime}\right)-\lambda \varphi_{1}(z)} \partial_{n_{x}} w_{\varepsilon}\left(t, x, y^{\prime}\right)\right|_{x \in \partial \Omega} \\
& =\left.\iint \partial_{n_{x}} f(t, x ; z, \lambda) w_{\varepsilon}\left(t, x, y^{\prime}\right)\right|_{x \in \partial \Omega} d t d x^{\prime}+O\left(e^{-C \lambda}\right) .
\end{aligned}
$$

Since for $t>t_{0}-\delta+\varepsilon$ or $x \in \Omega$

$$
\left(\partial_{t}^{2}-\Delta_{x}\right)\left[K_{+}\left(t, x, y^{\prime}\right)-w_{\varepsilon}\left(t, x, y^{\prime}\right)\right] \equiv 0,
$$

and $K^{+}$and $w_{\varepsilon}$ have no singularities for $t<0$, we deduce from the strict convexity of $\partial \Omega$ that, for $t>t_{0}-\delta+\varepsilon$, we have

$$
\begin{gathered}
p\left(t ; y_{0}^{\prime}, \eta_{0}^{\prime}\right) \notin S S\left[\left.\left(K^{+}-w_{\varepsilon}\right)\right|_{x \in \partial \Omega^{+}}\right] \\
p\left(t ; y_{0}^{\prime}, \eta_{0}^{\prime}\right) \notin S S\left[\left.\partial_{n_{x}}\left(K^{+}-w_{\varepsilon}\right)\right|_{x \in \partial \Omega^{+}}\right] .
\end{gathered}
$$


Therefore in the left hand side of (5.23), we can replace $\partial_{n} w_{\varepsilon}$ by $k^{+}\left(t, x^{\prime}, y^{\prime}\right)$. Since

$$
\left.w_{\varepsilon}\right|_{x \in \partial \Omega}=\mathbf{T}^{1}\left(\theta_{\varepsilon} k^{+}\right) \text {, }
$$

the relation

$$
k^{+} \in I_{A^{+}, \psi}^{3} \text { for } t<t_{0}
$$

implies that $\left.w_{\varepsilon}\right|_{x \in \partial \Omega}$ is microlocally Gevrey 3 with weight $\psi+o(1)$ near $p\left(t_{0}-\delta_{0}, y_{0}^{\prime}, \eta_{0}^{\prime}\right)$, with $o(1)$ going to zero with $\varepsilon$ (use Lemma 4.2). From (5.24), we deduce that $w_{\varepsilon}$ is microlocally zero near the points $p\left(t ; y_{0}^{\prime}, \eta_{0}^{\prime}\right)$ if $t>t_{0}-\delta_{0}+\varepsilon$. To conclude the proof, we estimate $\partial_{n} f\left(t, x^{\prime}, z, \lambda\right)$ for $z$ near $z_{1}=\pi \circ \chi_{1}\left(q_{0}^{(a)}\right), t$ near $t_{0}-\delta_{0}$ and $x^{\prime}$ near $\chi^{\prime}\left(t_{0}-\delta_{0} ; y_{0}^{\prime},-\eta_{0}^{\prime}\right)$. Using (5.19) and (5.20), we obtain:

$$
e^{i \lambda \varphi_{1}(z)} \partial_{n} f\left(s, y^{\prime}, z, \lambda\right)=e^{i \lambda \Phi\left(s, y^{\prime}, z\right)-\lambda^{1 / 3} \psi\left(s, y^{\prime}, z\right) \sigma\left(s, y^{\prime}, z, \lambda\right)} .
$$

In the right hand side, $\Phi\left(s, y^{\prime}, z\right)$ denotes the holomorphic phase which describes the lagrangian manifold

$$
\left(\chi_{1} \otimes \mathrm{Id}\right)\left[\left(t, x^{\prime}, \tau, \xi^{\prime}\right)=\exp l H_{\mathfrak{\tau}-\left|\xi^{\prime}\right|}\left(s, y^{\prime},-\sigma,-\eta^{\prime}\right) ; \quad \tau=\left|\xi^{\prime}\right|, \sigma=-\left|\eta^{\prime}\right|\right] .
$$

$\psi$ is given by integrating the one-form $e^{i \pi / 6} 2^{-1 / 3} J$ from $\alpha$ to $\beta$ along a leaf of $G^{\mathbb{C}}$ where $\alpha$ and $\beta$ are given by

$$
\alpha=\left(z, \Phi_{z}^{\prime}\left(s, y^{\prime}, z\right)\right), \quad \beta=\left(s, y^{\prime},-\Phi_{s, y}^{\prime}\left(s, y^{\prime}, z\right)\right)
$$

with an implicit identification by the isomorphism $\chi_{1} \otimes \operatorname{Id}$. The symbol $\sigma\left(s, y^{\prime}, z, \lambda\right)$ is $C^{\omega}$ and classical with respect to $\lambda^{1 / 3}$. To complete the proof, one estimates the right hand side of (5.23). This is done using the fact that $w_{\varepsilon}$ is microlocally Gevrey 3 with weight $\operatorname{Re} \psi+o(1)$ near the point $p\left(t_{0}-\delta ; y_{0}, \eta_{0}\right)$ and the inversion formula for the F.B.I. transform (cf. Fig. 2).

Fig. 2

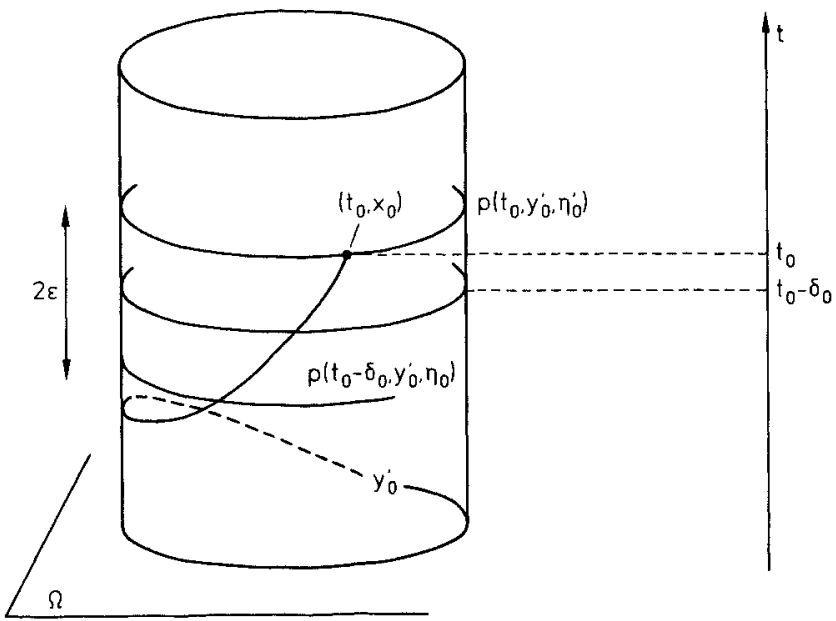


Lemma 5.3. With notations from Lemma 5.2, we suppose that $f$ is $\mathrm{V}^{+}$.m.p. $\mathrm{G}^{3}$ on $V^{+}$ and microlocally Gevrey 3 with weight $m^{\prime}$ at $p_{0}$ with $m^{\prime}\left(p_{0}\right)>m\left(p_{0}\right)$. Let $F_{0} \subset V^{+}$be the bicharacteristic through $p_{0}$. Then there is a weight $m^{\prime \prime}>m$ such that $f$ is microlocally Gevrey 3 on $F_{0}$ with weight $m^{\prime \prime}$.

Proof. Since the Definition 5.2 does not depend on the F.B.I. transform choosen, we can work in the standard setting:

$$
\begin{gathered}
z=\left(z^{\prime}, z^{\prime \prime}\right), \varphi=(\operatorname{Im} z)^{2} / 2, \quad V^{+}=\left\{\operatorname{Im} z^{\prime}=0\right\}, \varphi_{V}=\left(\operatorname{Im} z^{\prime \prime}\right)^{2} / 2, \\
F_{0}=\left\{\left(z^{\prime}, 0\right) \mid \operatorname{Im} z^{\prime}=0\right\} .
\end{gathered}
$$

We consider a real $C^{\omega}$ weight function $m\left(z^{\prime}, z^{\prime \prime}\right)$ pluriharmonic with respect to $z^{\prime}$.

Let $f(z ; \lambda)$ be a holomorphic function which satisfies the two estimates

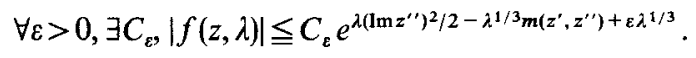

For

$$
|z| \leqq \varrho_{0},|f(z, \lambda)| \leqq e^{\lambda(\operatorname{Im} z)^{2} / 2-C_{0} \lambda^{1 / 3}}, \quad \text { with } \quad C_{0}>m(0,0) .
$$

The maximum principle implies the existence of two positive constants $\varrho_{1}>0$ and $C_{1}>m(0,0)$, such that:

$$
|f(z, \lambda)| \leqq e^{\lambda\left(\operatorname{Im} z^{\prime \prime}\right)^{2} / 2-C_{1} \lambda^{1 / 3}} \forall z,|z| \leqq \varrho_{1} .
$$

Let $G\left(z^{\prime}, z^{\prime \prime}\right)$ be a function holomorphic with respect to $z^{\prime}$ which satisfies $\operatorname{Re} G=m$. Let $F$ be the function

$$
F\left(z^{\prime}, z^{\prime \prime}, \lambda\right)=f\left(z^{\prime}, z^{\prime \prime} ; \lambda\right) e^{\lambda^{1 / 3} G\left(z^{\prime}, z^{\prime \prime}\right)}
$$

Then,

$$
\forall \varepsilon>0, \exists C_{\varepsilon}, \quad|F| \leqq C_{\varepsilon} e^{\lambda\left(\operatorname{Im} z^{\prime \prime}\right)^{2} / 2+\varepsilon \lambda^{1 / 3}},
$$

and

$$
|F| \leqq e^{\lambda\left(\operatorname{Im} z^{\prime \prime}\right)^{2} / 2-C_{2} \lambda^{1 / 3}} \quad \text { with } C_{2}>0 \text { for }|z|<\varrho_{2} .
$$

Since $F$ is holomorphic with respect to the variable $z^{\prime}$ a second application of the maximum principle shows that (5.25) and (5.26) suffice to complete the proof of the Lemma 4.3.

The next proposition passing from local to global in time is a major step in our proof.

Proposition 5.3. For any positive t the kernel $k^{+}$belongs to the class $I_{A^{+}, \psi}^{3}$

Proof. We have to show that $k^{+}$belongs to the class $I_{A^{+}, \psi}^{3}$ near the points $p\left(t_{0} ; y^{\prime}, \eta^{\prime}\right), t>0,\left(y^{\prime}, \eta^{\prime}\right) \in T^{*} \partial \Omega$.

Near $p\left(t ; y_{0}, \eta_{0}\right), t<t_{0}$, we have $k^{+}=k^{+, 1}+k^{+, 2}$ where $k^{+, 2}$ is microlocally Gevrey 3 with weight $m>\operatorname{Re} \psi$ and $k^{+, 1}$ has a representation as in (3.6). From the definitions of $k^{+}, \mathbb{T}^{\mathbf{1}}$, we see that $k^{+}$satisfies the hypothesis of the Proposition 4.1 and that $\mathbb{T}^{1}\left(k^{+}\right)=0$ microlocally near $A$ for $t>0$ so if $k^{+}$is the symbol of $k^{1,+}$ we have

$$
\widehat{T}^{1}\left(\hat{k}^{+}\right) \equiv 0 \text {. }
$$


From Proposition 4.2, we deduce that the formal operator $\hat{\mathbb{T}}^{1}$ has simple characteristics on $V^{+}$(it is analytic-classical with large parameter $\lambda^{1 / 3}$ instead of $\lambda$ ), therefore $\hat{k}^{+}$can be extended to a neighborhood of $t_{0}$ and therefore we may assume that $k^{+, 1}$ is also defined in a neighborhood of $t_{0}$. By Lemma $5.2, k^{+}$is $\mathrm{V}^{+}$.m.p. $\mathrm{G}^{3}$ near $p\left(t_{0} ; y_{0}^{\prime}, \eta_{0}^{\prime}\right)$. Since $k^{+, 1}$ belongs to $I_{A^{+}, w}^{3}$, it is also V.m.p. $G^{3}$ near the same point. Therefore, the same property holds for $r=k-k^{+, 1}$.

Applying Lemma 5.3 to $r$ completes the proof of Proposition 5.3.

Remark 5.3. Except for the formula (2.13), where they are used to reduce the problem to $\partial \Omega$, the proof of the Proposition 5.3 is the only place where the operators $\mathbb{T}^{i}$ play an essential role.

\section{Proof of the Theorem 3}

We recall that $\operatorname{Tr}(t)$ is for $t>0$ a $C^{\infty}$ function, given by the formulas (2.12), (2.13):

$$
-\operatorname{Tr}(t)=2(\partial / \partial t) \int B(t, x, x) d x
$$

with $B(t, x, y)$ given by

$$
B(t, x, y)=\int_{-\infty}^{\infty} d s \int_{\partial \Omega} \mathbb{T}^{2}(t-s, x, z) k^{+}(s, z, y) d z .
$$

Since we have proven, in Proposition 5.2, that $k^{+}$belongs to $I_{A, \psi}^{3}$ in $t>0$, we deduce from Proposition 4.1 that in $t>0,|x-y|<t$

$$
S S(B) \subset A
$$

and

$$
B \in I_{\Lambda, \psi}^{3}
$$

Choose $\varepsilon$ so that $\{|t-T|<\varepsilon\}$ contains only $T$ from the length spectrum of $\partial \Omega$. We deduce from (4.2), and part (i) of Theorem 3 that in a neighborhood of $t=T$ we that we have.

$$
S S(\operatorname{Tr}(t)) \subset\{(t, \tau), \exists(x, \xi),(t, \tau, x, \xi, x,-\xi) \in \Lambda\}
$$

and this proves part (ii).

Now suppose that $\gamma$ is a closed geodesic of length $T_{0}$ which satisfies the hypothesis of part (iii). We use the arc length $s$ as a parameter on $\gamma$. The lift of $\gamma$ in $T^{*}(\partial \Omega)$ is the union of the two bicharacteristics curves

$$
\Gamma^{ \pm}=\{x=\gamma(s), \xi= \pm k(d \gamma / d s), k>0\} .
$$

Proposition 6.1. For $t$ near $T_{0}$, one has $\operatorname{Tr}(t) \in I_{\Lambda_{0}, \psi_{0}}^{3}$ with

$$
\Lambda_{0}=T_{T_{0}}^{*}(\mathbb{R}) ; \quad \psi_{0}\left(T_{0}, \tau\right)=|\tau|^{1 / 3} e^{i \pi \operatorname{sign}(\tau) / 6} 2^{-1 / 3} \omega \int_{0}^{T_{0}} \varrho^{2 / 3}(s) d s
$$


in addition, microlocally near $\left(T_{0},+1\right)$, one has

$$
\operatorname{Tr}(t)=\int_{1}^{\infty} e^{i\left(t-T_{0}\right)-\psi_{0}\left(T_{0}, \tau\right)} a(z) d z+r(t),
$$

where $a$ is an analytic symbol in $\lambda^{-1 / 3}$ of degree zero and $r(t)$ is microlocally Gevrey 3 with weight strictly greater than $\operatorname{Re} \psi_{0}\left(T_{0}, z\right)$ near $\left(T_{0},+1\right)$.

Proof. Since $\operatorname{Tr}(t)$ is real, it is enough to consider the case $\tau>0$. Furthermore, for any $(x, \xi) \in \Gamma^{ \pm}$, one has $\psi(T, x, \xi)=\psi_{0}(T, \xi \mid)$. Since $\gamma$ is non degenerate and since $T_{0}$ is isolated, the fixed points of the mapping

$$
\exp -T_{0} H_{|\xi|}: T^{*} \partial \Omega \backslash 0 \rightarrow T^{*} \partial \Omega \backslash 0
$$

are the points of $\Gamma^{ \pm}$and these fixed points are clean in the sense of [D.G.]. Therefore the principal part of the right hand side of (2.12) can be computed, for $(t, \tau)$ near $(T, 1)$, by the classical method of [D.G.], [C.], provided the computation of (7.5) in [D.G.] is replaced by the following lemma.

Lemma 6.1. Let $f(s, x)$ be an analytic function defined for $s \in S^{1}$ and $x \in \mathbb{R}^{p},|x|<r$ and let $l(s, x)$ be an analytic function which satisfies

$$
l(s, 0)=C^{t e} \equiv l_{0} .
$$

Suppose that $f$ satisfies

$$
\begin{gathered}
\operatorname{Re} f(s, x)>0 \text { for } \quad|x| \neq 0, f(s, 0) \equiv 0, \nabla f(s, 0) \equiv 0 . \\
f \text { is transversally non degenerate on } S^{1} \times\{0\} .
\end{gathered}
$$

Then, for any analytic symbol, $a(s, x, \lambda)$, classical in $\lambda^{1 / 3}$ and of degree $d$, the integral

$$
I_{a}(\lambda) \equiv{ }_{s^{1} \times\{|x| \leqq r\}} e^{-\lambda f(s, x)-\lambda^{1 / 3}[(s, x)} a(s, x, \lambda) d s d x,
$$

is equal to $e^{-\lambda^{1 / 3} l_{0}} b(\lambda)$ where $b(\lambda)$ is an analytic symbol, classical in $\lambda^{1 / 3}$, of degree $d-p / 2$. Furthermore, the principal symbol is given by

$$
b^{0}(\lambda)=(2 \pi)^{p / 2} \int_{s^{1}} \theta(s) a^{0}(s, 0) d s, \theta(s) \equiv\left(\operatorname{det} f_{x x}^{\prime \prime}(s, 0)\right)^{-1 / 2},
$$

where the square root is chosen to converge to 1 when u goes to zero in the homotopy

$$
u \rightarrow(1-u) \mid d+u f_{x x}^{\prime \prime} .
$$

The proof of this lemma, using the Morse lemma and the method of steepest descent is ommitted. Proposition 6.1 is a straightforward consequence.

Proposition 6.2. The principal part $a_{0}$ of the symbol $a(\tau)$ in (6.3) is not equal to zero. Proof. The principal symbol $a_{0}$ is the sum of two terms $a_{0}=a_{0}^{+}+a_{0}^{-}$, where $a_{0}^{ \pm}$ corresponds to the contribution of $\Gamma^{ \pm}$. We know that for $t>0$ the kernel $k^{+}(t, x, y)$ belongs to $I_{A, \psi}^{3}$. We denote by $k^{+, 0}(t, x, \xi)$ the leading term of its symbol (which is of 
degree 1), when it is written in the form (4.11).

$$
k^{+}(t, x, y) \simeq \int e^{i \varphi(t, x, y, \xi)-l(t, x, y, \xi)} \hat{k}^{+}(t, x, \xi) d \xi,
$$

with phase $\varphi$ defined by

$$
\partial_{t} \varphi=\Theta(x, x, \partial \varphi / \partial x), \quad \varphi(0, x, y)=(x-y) \cdot \xi .
$$

From Proposition 5.1, we deduce that the mapping $t \rightarrow k^{+, 0}(t, x, \xi)$ can be extended to $t=0$ and that we have $k^{+, 0}(0, x, \xi) \in i \mathbb{R}_{+}^{*}$.

Since we have, for $t>0, \hat{\mathbf{T}}^{1} k=0$, Proposition 4.2 (and in particular the formula (4.24)) implies that $k^{+, 0}$ is constant along the geodesic flow. Finally, using formula (4.24), $B=\mathbb{T}^{2} \cdot k$ and the method of [D.G.] for the computation of the principal symbol of the function $B=\int B(t, x, x) d x$, yields $a_{0}^{ \pm}=\alpha^{ \pm} i^{\sigma^{*}}$, where $\alpha^{ \pm}$denote two non zero complex numbers with the same argument while $\sigma_{ \pm}$are the Maslov indices related to the curves $\Gamma^{ \pm}$. The proof is therefore a consequence of the equality $\sigma^{+}=\sigma^{-}$which is proved in the Appendix 1.

We are now ready to prove the final assertion (2.5) of Theorem 3. If (2.5) were not true there would exist $\varepsilon>0$ and $\eta>0$ such that one has both:

$$
\varlimsup_{n \rightarrow \infty} \sup _{|t-T|<\varepsilon}\left|\frac{1}{3 n !} \frac{d^{n} \operatorname{Tr}(t)}{d t^{n}}\right|^{1 / 3 n} \leqq\left(C_{\gamma} T+\varepsilon\right)^{-1}
$$

and

$$
\varlimsup_{n \rightarrow \infty} \sup _{|t-T|<\varepsilon}\left|\frac{1}{3 n !} \frac{d^{n} r(t)}{d t^{n}}\right|^{1 / 3 n} \leqq\left(C_{\gamma} T+\varepsilon\right)^{-1} .
$$

Choose $\chi \in$ Gevrey $^{2}(\mathbb{R})$, Supp $\chi \subset\left\{\left|t-T_{0}\right|<\varepsilon\right\}, \chi \equiv 1$ near $T_{0}$. Then $f(t) \equiv \chi(t) \operatorname{Tr}(t)$ satisfies

$$
\varlimsup_{n \rightarrow \infty} \sup _{\mathbb{R}}\left|\frac{1}{3 n !} \frac{d^{n} f(t)}{d t^{n}}\right|^{1 / 3 n} \leqq\left(C_{\gamma} T+\varepsilon\right)^{-1}
$$

In addition, for $z \in \mathbb{C}$ near $T_{0}-i$, one has with a $C>0$,

$$
\widetilde{f}(z, \lambda) \equiv \int e^{-\lambda(z-t)^{2} / 2} f(t) d t=\int_{1 T_{0}-\varepsilon, T_{0}+\varepsilon[} e^{-\lambda(z-t)^{2} / 2} \operatorname{Tr}(t) d t+O\left(e^{-C \lambda}\right) .
$$

The integral defining $f$ is equal to

$$
(2 \pi \lambda)^{-1 / 2} \int e^{i z z-\tau^{2} / 2 \lambda} \hat{f}(\tau) d \tau
$$

and using (6.6),

$$
|\hat{f}(\tau)| \leqq A((3 n) !) /\left(\tau^{n}\left(C_{0} T_{0}+\eta\right)^{3 n}\right)
$$

which in turn implies

$$
\left|f\left(z_{0}, \lambda\right) e^{-\lambda / 2}\right| \leqq A^{\prime \prime} e^{-\beta \lambda^{1 / 3}}, \quad \beta>C_{0} T_{0} .
$$


On the other hand, from (6.3), one has as $\lambda \rightarrow \infty$

$$
e^{-\lambda / 2} f(z, \lambda) e^{\lambda^{1 / 3} \psi_{0}\left(T_{0}, l\right)} \sim a_{0} \neq 0 .
$$

Since $\operatorname{Re} \psi_{0}\left(T_{0}, l\right)=C_{0} T_{0}$, the contradiction between (6.7) and (6.8) completes the proof.

\section{Appendix 1}

Proposition 1. Let $\Gamma$ be a closed geodesic on $M=\partial \Omega$ and let

$$
\Gamma^{ \pm}=\{x=\gamma(s), \xi= \pm d \gamma / d s\} \subset T^{*}(M)
$$

be the two bicharacteristics projecting on $\Gamma$, parametrised by the arclength. Then the Maslov indices $\sigma_{+}$and $\sigma_{-}$related to the curves $\Gamma^{+}$and $\Gamma^{-}$coincide.

Proof. Starting from $x_{0}=\gamma(0)=\gamma(T)$, we define the phase function: $\varphi^{+}(x, y, \xi, t)$ by the eiconal equation:

$$
\partial_{t} \varphi=\left|\varphi_{x}^{\prime}\right|
$$

and the initial condition:

$$
\varphi(x, y, \xi, 0)=(x-y) \cdot \xi .
$$

The Eq. (A.1) is defined in the complementary of the focal points $\left(x_{1}, x_{2}, \ldots, x_{N}\right)$ which are of finite number. At each focal point $x_{p}, v_{p} \pi / 2$ is added to the phase $\varphi$. Since the Maslov index is an integer, it is enough to prove the result for a sligthly perturbed hamiltonian on $\partial M$. With this perturbation, we may assume that $\gamma(T)$ is not a focal point. We denote by $\varphi_{p}$ the phase on the $\operatorname{arc}\left(x_{p}, x_{p+1}\right) . \varphi_{p}$ is a real non degenerate phase; and from 0 to $T$, microlocally near $\left(\gamma(0), \gamma^{\prime}(0)\right)$, the identity

$$
\int e^{i(x-y) \xi} d \xi
$$

is changed into the operator

$$
i^{v_{1}+v_{2}+\ldots v_{p}} \int e^{i\left(\varphi_{N}(x, \xi)-y \cdot \xi\right)} b_{+} d \xi,
$$

where $b_{+}$, constructed by the transport equation, is a real positive symbol.

Now we notice that, since $\varphi_{N}(x, \xi, T)$ is a real non degenerate phase, a microlocal identity is given by

$$
\mathbb{T}=\int e^{l\left(\varphi_{N}(x, \xi)-\varphi_{N}(y, \xi)\right)} b d \xi,
$$

microlocally near $\left(\gamma(0)=\gamma(T), \gamma^{\prime}(0)=\gamma^{\prime}(T)\right)$, where $b$ is a real positive symbol. We use this form of the identity to construct a microlocal parametrix on $\Gamma^{-}$, starting from $\left(\gamma(0),-\gamma^{\prime}(0)\right)$, this changes the exponential appearing in $T$ into its conjugate,

$$
\mathbf{T}=\int e^{\left.i \varphi_{N}(y, \xi)-i \varphi_{N}(x, \xi)\right)} b d \xi .
$$


Reversing the time, we see that passing through any focal point $x_{p}$ introduces a factor $i^{-v_{p}}$. Therefore

$$
e^{-i \varphi_{N}(x, \xi)}=e^{i \varphi_{N}(x, \xi)}
$$

is changed into $i^{v_{1}+v_{2}+\ldots v_{p}} e^{-i x \xi}$.

Thus we have shown that on $\Gamma^{+}$the identity is changed into:

$$
\mathbb{T}^{+}=i^{v_{1}+v_{2}+\ldots v_{p}} \int e^{i \varphi_{N}(x, \xi)-i y \xi} b_{+} d \xi
$$

and that on $\Gamma^{-}$, it is changed into:

$$
\mathbb{T}^{-}=i^{v_{1}+v_{2}+\ldots v_{p}} \int e^{i \varphi_{N}(y \cdot \xi)-i x \xi} b_{-} d \xi,
$$

Taking $x=y$ in the right hand side of (A.4) and (A.5) and integrating over $x$ gives the result.

\section{Appendix 2}

In this appendix, we briefly discuss the genericity of the geometric hypothesis of Theorem 2. Denote $M=\partial \mathcal{O}, S^{*} M=T^{*} M \backslash \mathcal{O} / \mathbb{R}_{+}$the cosphere bundle of $M$ and $\varphi_{t}$ the geodesic flow on $S^{*} M$.

Proposition 1. The condition of Theorem 2 is open in the sense that if $\gamma$ is a closed geodesic satisfying the hypotheses (1), (2) of Theorem 2, then all $C^{\infty}$ obstacles $C^{4}$ close to $\partial \mathcal{O}$ possess a closed geodesic $\gamma$ close to $\gamma$ satisfying the same conditions.

Proposition 2. The conditions of Theorem 2 are satisfied for a dense set of $M$ in the following sense. Any $M$ has a closed geodesic $\gamma$ and for any such $\gamma$ and any $k \geqq 4$ there are analytic manifolds arbitrarily close to $M$ in the $C^{k}$ topology with a closed geodesic close to $\gamma$ satisfying the conditions of Theorem 2.

Sketch of Proof of Proposition 1. Let $\gamma_{ \pm}$be the two liftings of $\gamma$ to $S^{*} M$. Since 1 is a simple eigenvalue of the differential of the Poincaré map; a perturbation theorem of Poincare implies that nearby obstacles have nearby closed geodesics $\tilde{\gamma}$ with period and first return map close to those of $\gamma$. In addition, there are no other geodesics of $\tilde{M}$ which are close to $\tilde{\gamma}$.

On the other hand, compactness implies that given an open neighborhood of $\gamma_{+} \cup \gamma_{-}$in $S^{*} M$, there is a $\delta>0$ so that $\left|\varphi_{T}(p)-p\right| \geqq \delta$ for $p$ outside that neighborhood and thus for $\tilde{M}$ close to $M$ there are no closed geodesics of length near $T$ which lie close to this neighborhood. Combining the above two observations yields the proposition.

Sketch of proof of Proposition 2. It is well known that by a small perturbation of $M$ we can insure that the non degeneracy condition (2) is satisfied. We therefore suppose that (2) is satisfied and consider (1).

We construct perturbed analytic manifolds $M_{+}$as follows. For $f \in C^{\infty}(M, \mathbb{R})$ small in $C^{4}$ norm, one defines

$$
M_{f}=\{x+f(x) n(x): x \in M\},
$$


where $n(x)$ is the outward unit normal to $M$. We consider those $f$ such that

$$
\forall x \in \gamma, \forall|\alpha| \leqq 2, D^{\alpha} f(x)=0 .
$$

Since $\left.f\right|_{\gamma}=0$, one sees that $\gamma \in M_{f}$. Since $\left.d f\right|_{\gamma}=0$, we see that for $x \in \gamma, n(x)$ is also normal to $M_{f}$. Since $\gamma$ is geodesic in $M$, the osculating plane of $\gamma$ is spanned by its tangent and $n(x)$. As $n$ is also the normal to $M_{f}, \gamma$ is also geodesic in $M_{f}$, closed and of length $T$. Since $\left.D^{2} f\right|_{\gamma}=0$, we see that $\gamma$ satisfies the non degeneracy condition (2) of Theorem 2.

We denote $\varphi_{t}^{f}$ the geodesic flow in $S^{*} M_{f}$. We identify $S^{*} M_{f}$ and $S^{*} M$ by the parametrization (A.6). Let $B$ be the closed subspace of $C^{k}(M)$ consisting of functions satisfying (A.7), and let $B_{\varepsilon}=\{f \in B:\|f\| \leqq \varepsilon\}$.

We choose $\varepsilon$ so small that $M^{f}$ is a regular convex obstacle satisfying condition (1) for all $f \in B_{\varepsilon}$. We will show that there exist arbitrarily small $f \in B_{\varepsilon}$ such that $M^{f}$ also satisfies condition (1).

As in the proof of Proposition 1, there is a neighborhood $\omega$ of $\gamma_{+} \cup \gamma_{-}$in $S^{*} M$, and a $\delta>0$ such that for $\varepsilon$ sufficiently small, $f \in B_{\varepsilon}, p \in \partial \omega,|t-T|<\delta$, we have $\left|\varphi_{t}^{f}(p)-p\right|>\delta$. We must find arbitrarily small $f$ such that this is true for $p \in S^{*} M^{f} \backslash \omega \equiv K$ with, perhaps, a smaller $\delta$.

The crucial step is to show that for any $q \in K$, the point $\varphi_{T}^{0}(q)$ can be perturbed in any direction along $S^{*} M$ by suitable small $f$.

Lemma. For $q \in K$ and any vector $u$ tangent to $S^{*} M$ at $\varphi_{T}^{0}(q)$ there is an $f \in B$ such that

$$
\left.d_{s} \varphi_{T}^{s f}(q)\right|_{s=0}=u .
$$

Assuming the lemma, we complete the proof of Proposition 2. Since $K$ is compact, we can find a finite dimensional linear subspace $F$ of $B$ with the property that for any $q \in K$ the differential at $f=0$ of the map $B \ni f \rightarrow \varphi_{T}^{f}(q) \in S^{*} M$ is surjective. Thus, shrinking $\varepsilon$ if necessary, we see that in $S^{*} \times S^{*} \times F$ the subvarieties

$$
A \equiv\left\{(q, q, f): q \in K, f \in B_{\varepsilon} \cap F\right\} \quad \text { and } \quad C \equiv\left\{\left(q, \varphi_{T}^{f}(q), f\right): q \in K, f \in B_{\varepsilon} \cap F\right\},
$$

with $\operatorname{dim} A=\operatorname{dim} C=\operatorname{dim} S^{*}+\operatorname{dim} F$ intersect transversally. Thus either $A \cap C$ is empty or $A \cap C$ is a submanifold of dimension equal to $\operatorname{dim} F$. In the first case, $\gamma \in M=M_{0}$ satisfies the hypotheses of Theorem 2. In the second case, we consider the projection $\pi$ of $A \cap C$ on $F$.

If $(q, q, f) \in A \cap C$, then the same is true of $\left(\varphi_{t}^{f} q, \varphi_{t}^{f} q, f\right)$ so if $\delta q$ is the tangent to the geodesic $\varphi_{t}^{f}(q)$ at $t=0$ the $(\delta q, \delta q, 0)$ is tangent to $A \cap C$. Thus $\pi_{*}\left(T_{(q, q, f},(A \cap C)\right)$ has dimension at most $\operatorname{dim} F-1$ in $T_{f}(F)$. The theorem of Sard implies that $\pi(A$ $\cap C)$ is nowhere dense in $F$. Thus, we may choose $f \in F$ arbitrarily small so that $f$ $\notin \pi(A \cap C)$. For such $f, \gamma \in M_{f}$ satisfies condition (A.2) of Theorem 2. It remains to prove the lemma.

Sketch of Proof of Lemma. Fix $q \in K$ and let $\bar{x}, \bar{u}=\varphi_{T}^{0}(q) \in S^{*} M$. In local coordinates a product of balls in $\mathbb{R}^{n},|\bar{x}-x| \leqq r,|u-\bar{u}| \leqq r$, defines a small neighborhood of $\bar{x}, \bar{u}$. We will construct $f$ with support in $B(r)$, the subset of $B$ consisting of functions supported in $|x-\bar{x}| \leqq r$. We must show that the differential 
Fig. 3

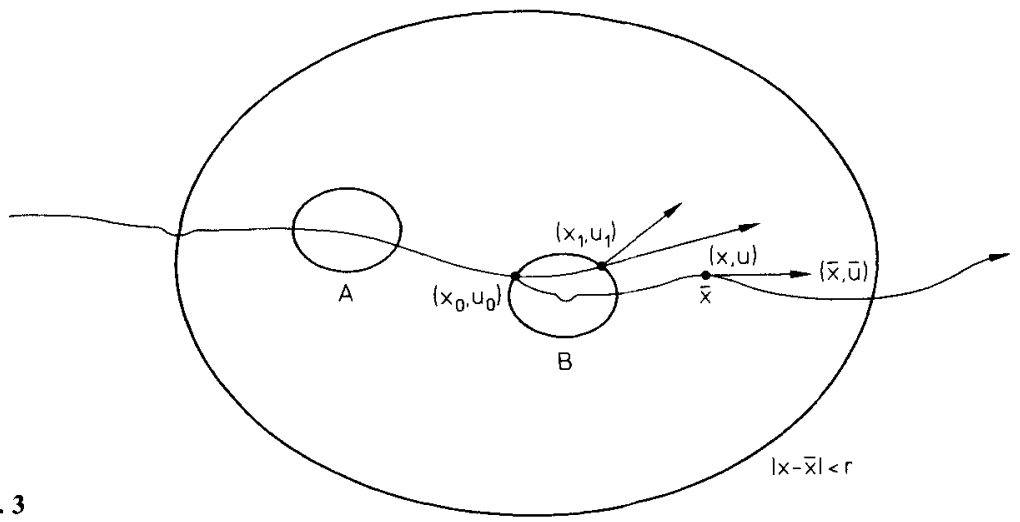

of the map

$$
B(r) \ni f \rightarrow \varphi_{T}^{f}(q) \in S^{*} M
$$

is surjective. It suffices to show that there is an $\left.\left.r_{1} \in\right] 0, r\right]$ and $C>0$ such that for all $x, u$ with $|x-\bar{x}|<r_{1},|u-\bar{u}|<r_{1}$, there is an $f \in B(r)$ with

$$
\varphi_{T}^{f}(q)=(x, u), \quad\|f\| \leqq C(|x-\bar{x}|+|u-\bar{u}|) .
$$

Choose discs in $M$ as in Fig. 3. We will construct $f$ with support in $A \cup B$. From $(x, u)$ as above trace the backward geodesic till it intersects $\partial B \times|u-\bar{u}| \leqq r$ at $\left(x_{1}, u_{1}\right)$. Let $x_{0}, u_{0}$ be the point in $\varphi_{t}(q)$ where $\gamma$ enters $B$. Choose a smooth curve on $M$ near $\gamma$ joining $x_{0}$ to $x_{1}$ and with cotangent passing from $u_{0}$ to $u_{1}$. For any $k$ this can be done with the $C^{k}$ distance to $\gamma$ being $O(|(x-\bar{x}, u-\bar{u})|)$. Choose $f$ of the same order, supported in $B$ so that this curve is a geodesic to $M$. To do this, one needs only deform $M$ so that the normal to $M$ is transformed to the principal normal of the curve. Having done this, the geodesic on the deformed $M$ arrives at $x, u$ in time $\widetilde{T}$ with $\tilde{T}-T=O(|x-\bar{x}, u-\bar{u}|)$. Next deform $M$ by choosing $f$ in $A$ so as to speed up or slow down the time spent through $A$ to compensate for the time lag. Here it is crucial that $M$ is strictly convex in $A$. The construction is complete.

\section{References}

[B.G.R.] Bardos, C., Guillot, J.-C., Ralston, J.: La relation de Poisson pour l'équation des ondes dans un ouvert non borné. Application à la théorie de la diffusion. Commun. Partial Differ. Equations 7, (8) 905-958 (1982)

[B.L.R.] Bardos, C., Lebeau, G., Rauch, J.: Méthodes semi classiques en mécaniques quantique. 1984. Publications de l'Université de Nantes

[BdM.] Boutet de Monvel, L.: Séminaire. Opérateurs Pseudodifférentiels Analytiques, Grenoble, 1975

[C.] Chazarain, J.: Formule de Poisson pour les variétés riemaniennes. Invent. Math. 24, 65-82 (1974)

[D.G.] Duistermaat, J.-J., Guillemin, V.: The spectrum of positive elliptic operators and periodic geodesics. Invent. Math. 24, 39-80 (1975) 
[G.] Goodhue, W.: Scattering theory for hyperbolic systems with coefficients of Gevrey type. Trans. Am. Math. Soc. 180, 337-346 (1973)

[H.1] Hörmander, L.: Fourier integral operators. I. Acta Math. 127, 79-183 (1971)

[H.2] Hörmander, L.: The Analysis of linear partial differential operators 1. Lect. Notes Math., Vol. 256. Berlin-Heidelberg-New York: Springer 1983

[L.] Lax, P.D.: Asymptotic solutions of initial value problems. Duke Math. J. 24, 627-646 (1957)

[LP.1] Lax, P.D., Phillips, R.S.: Scattering theory. New York: Academic Press 1967

[LP.2] Lax, P.D., Phillips, S.R.: A logarithmic bound on the location of the poles of the scattering mat. Arch. Rat. Mech. Anal. 40, 268-280 (1971)

[L.1] Lebeau, G.: Deuxième microlocalisation sur les sous-variétés isotropes. Ann. Inst. Fourier 35, (2) 145-216 (1985)

[L.2] Lebeau, G.: Régularité Gevrey trois pour la diffraction. Commun. Partial Differ. Equations 9, (15) 1437-1494 (1984)

[L.3] Lebeau, G.: Propagation Gevrey pour le problème mixte. Advanced in microlocal analysis, 1986 NATO ASI published by Reidel (Garnir editor)

[M.1] Melrose, R.: Singularities and energy decay in acoustical scattering. Duke Math. J. 46, (1) 43-59 (1979)

[M.2] Melrose, R.: Polynomial bound on the number of scattering poles. J. Funct. Anal. 53, 287-303 (1983); also Proc. of St. Jean de Monts 1984 seminar

[M.S.] Melrose, R., Sjostrand, J.: Singularities of boundary value problems I and II. Commun. Pure Appl. Math. 31, 595-617 (1978); 35, 129-168 (1982)

[P] Popov, G.: Estimates near the shadow and poles of the S. matrix. (Preprint)

[S.K.K.] Sato, M., Kawai, T., Kashiwara, M.: Microfunctions and pseudo differential equations. Lect. Notes Math., Vol. 287. Berlin-Heidelberg-New York: Springer 1973

[S.1] Sjöstrand, J.: Singularités analytiques microlocales. Astérisque 95, 1-166(1982)

[S.2] Sjöstrand, J.: Propagation of analytic singularities for second order Dirichlet problems. Commun. Partial Differ. Equations 5, (1) 41-94 (1980)

[R.S.] Rauch, J., Sjöstrand, J.: Propagation of analytic singularities along diffracted rays. Indiana Univ. Math. J. 30, (3) 389-401 (1981)

Oblatum 30-IX-1986 \& 17-XI-1986

\section{Note added in proof}

The authors want to thank Prof. V. Petkov for having pointed to them the following fact: The first indication of a polynomial bound (in the spirit of inequality (1.4)) for the resolvent set seems due Babic and Grigor'eva (cf. V. M. Babic and N. S. Grigor'eva: The analytic continuation of the resolvent of the exterior three dimensional problem for the Laplace operator to second sheet Funkcional Analysis $i$ Prilozen. 8. (1974) $\left.n^{\circ} 1,71-74\right)$. 